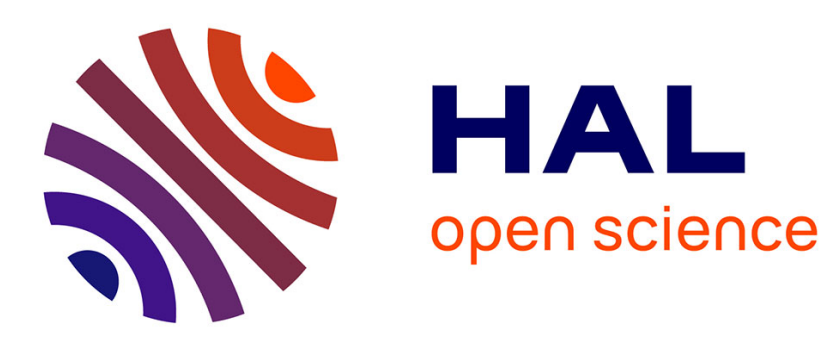

\title{
Numerical studies of porous ductile materials containing arbitrary ellipsoidal voids - II: Evolution of the length and orientation of the void axes
}

Komlanvi Madou, Jean-Baptiste Leblond, Léo Morin

\section{- To cite this version:}

Komlanvi Madou, Jean-Baptiste Leblond, Léo Morin. Numerical studies of porous ductile materials containing arbitrary ellipsoidal voids - II: Evolution of the length and orientation of the void axes. European Journal of Mechanics - A/Solids, 2013, 42, pp.490 - 507. 10.1016/j.euromechsol.2013.06.005 . hal-01436276

\section{HAL Id: hal-01436276 \\ https: / hal.sorbonne-universite.fr/hal-01436276}

Submitted on 16 Jan 2017

HAL is a multi-disciplinary open access archive for the deposit and dissemination of scientific research documents, whether they are published or not. The documents may come from teaching and research institutions in France or abroad, or from public or private research centers.
L'archive ouverte pluridisciplinaire HAL, est destinée au dépôt et à la diffusion de documents scientifiques de niveau recherche, publiés ou non, émanant des établissements d'enseignement et de recherche français ou étrangers, des laboratoires publics ou privés. 


\title{
Numerical studies of porous ductile materials containing arbitrary ellipsoidal voids - II: Evolution of the length and orientation of the void axes
}

\author{
Komlanvi Madou, Jean-Baptiste Leblond ${ }^{*}$, Léo Morin \\ UPMC Univ Paris 6 and CNRS, UMR 7190, Institut Jean Le Rond d'Alembert, F-75005 \\ Paris, France
}

\begin{abstract}
In Part I, Madou and Leblond (2012a,b)'s criterion for plastic porous materials containing arbitrary ellipsoidal voids was validated by comparing its predictions with the results of some numerical limit-analyses of elementary cells containing such voids. In the present Part II, our aim is now to complete the model by proposing reasonable evolution equations for the length and orientation of the axes of the voids. Again, however, the equations proposed are not attached to this specific model and could be used in conjunction with any similar criterion accounting for void shape effects.

In the definition of the evolution equations looked for, a central role is played by "elastic" expressions for the strain and rotation rates of the voids proposed by Ponte-Castaneda and Zaidman (1994) and Kailasam and Ponte-Castaneda (1998) from homogenization theory. The importance of plastic effects however makes it necessary to modify these expressions; this is done heuristically by introducing stress-dependent correction factors determined numerically in a number of reference cases and suitably interpolated between these cases.

Keywords: Porous ductile solids; ellipsoidal voids; numerical study; evolution of void shape and orientation
\end{abstract}

\section{Introduction}

Part I was devoted to the numerical validation of Madou and Leblond (2012a,b)'s recently proposed criterion for plastic porous materials containing arbitrary ellipsoidal voids, which stands as a generalization of the GLD criterion for spheroidal voids (Gologanu et al., 1993, 1994; Gologanu, 1997; Gologanu et al., 1997) itself extending Gurson (1977)'s famous criterion for spherical voids. The validation was based on numerical limit-analyses, performed by the finite element method, of a number of elementary ellipsoidal cells containing confocal ellipsoidal voids, as considered in the derivation of Madou and Leblond (2012a,b)'s criterion.

\footnotetext{
* Corresponding author.
} 
Our aim in the present Part II is now to propose reasonable evolution equations for the length and orientation of the axes of the voids, which act as internal parameters in Madou and Leblond (2012a,b)'s model. The definition of these equations will make a heavy use of the results of some numerical limit-analyses of hollow ellipsoidal cells analogous to those of Part I and performed with the same techniques, though with a different purpose.

It is again important to note that although our attention is focussed here on Madou and Leblond (2012a,b)'s model, the evolution equations that will be proposed are not in fact attached to this specific model and could be used to complete any model for plastic porous material containing arbitrary ellipsoidal voids, like that of Danas and Ponte-Castaneda (2008a,b) for instance. (Such a use, based on an implicit assumption of independence of the yield function and the void strain and rotation rates, would of course be acceptable only for models not based on some theory implying explicit specific relations between these quantities).

The paper is organized as follows:

- Section 2 presents the general form of the expression proposed for the void strain rate. An accurate expression for elastic voided materials derived by Ponte-Castaneda and Zaidman (1994) from homogenization theory is adopted as a reference. This expression is however modified through introduction of heuristic correction coefficients aimed at accounting for plastic effects, the importance of which has been evidenced in several previous studies, starting with the seminal work of Budiansky et al. (1982).

- Section 3 explains the determination of these coefficients. One first considers voids having the shape of a sphere, a circular cylinder or an infinite planar layer ("sandwich" case). The coefficients are determined numerically in the first two cases and noted to be unity in the third one. Interpolation formulae are then proposed between these cases, considering (i) a prolate spheroid as intermediate between a sphere and a circular cylinder; (ii) an oblate spheroid as intermediate between a sphere and an infinite planar layer; and (iii) a general ellipsoid as intermediate between prolate and oblate spheroids.

- Section 4 presents the expression proposed for the void rotation rate. The approach adopted is basically identical to that used for the void strain rate in that it consists in introducing heuristic corrections into an "elastic" expression proposed by Kailasam and Ponte-Castaneda (1998). It is however simpler in detail, because Kailasam and PonteCastaneda (1998)'s elastic expression is exact in both the spherical and sandwich cases, so that the correction coefficients (a single one, in fact) need be evaluated numerically in the sole circular cylindrical case.

- Section 5 relates the evolution of the length and orientation of the axes of the void to its strain and rotation rates. One numerical drawback of the standard expression of the rotation rate of the axes is that it diverges when two semi-axes become equal. This difficulty is circumvented by determining instead the evolution equation of the quadratic form characterizing the ellipsoidal void geometry, where no such divergence appears. The instantaneous length and orientation of the axes of the voids may then be obtained through diagonalization of this quadratic form at each instant.

- Section 6 finally summarizes the evolution equations proposed for ease of reference. 


\section{Expression of the strain rate of ellipsoidal voids - Generalities}

The first task is to find a good expression of the strain rate of the void. It has already been noted by Gologanu et al. (1993, 1994); Gologanu (1997); Gologanu et al. (1997) that doing so is more difficult than defining a reasonable approximate yield criterion. The reason is that the overall yield locus admits a variational characterization in terms of the overall plastic dissipation, whereas the void strain rate does not. As a consequence, if one adopts a trial velocity field differing modestly from the true one in an approximate limit-analysis of the elementary cell considered, one must get a good estimate of the minimum of this dissipation leading to a good estimate of the yield locus; but nothing warrants that the corresponding estimate of the void strain rate will be of similar quality. In practice, in the spheroidal case, it has been noted by Gologanu et al. (1993, 1994); Gologanu (1997); Gologanu et al. (1997), that Gologanu et al. (1993, 1994)'s trial velocity fields used in the derivation of the GLD criterion lead to poor predictions of the void strain rate. In the general ellipsoidal case, since Leblond and Gologanu (2008)'s fields used in the derivation of Madou and Leblond (2012a,b)'s criterion are just extensions of Gologanu et al. (1993, 1994)'s fields to the general ellipsoidal geometry, their use to estimate the void strain rate cannot be hoped to lead to better results.

For this reason, we shall drop here analytical limit-analysis based on Leblond and Gologanu (2008)'s fields. We shall use instead as a reference a well-established expression of the void strain rate for elastic porous materials proposed by Ponte-Castaneda and Zaidman (1994), and simply introduce heuristic correction factors determined numerically into this expression to account for plastic effects, the importance of which has long been well-known from the seminal work of Budiansky et al. (1982) and its many successors.

\subsection{Ponte-Castaneda and Zaidman's equation for the void strain rate and its suggested modification}

Ponte-Castaneda and Zaidman (1994)'s formula, derived from homogenization theory, for the strain rate tensor $\mathbf{D}^{v}$ of an ellipsoidal void embedded in an isotropic incompressible elastic body, reads

$$
\mathbf{D}^{v}=\mathbf{L}^{\mathrm{el}}: \mathbf{D} \quad, \quad \mathbf{L}^{\mathrm{el}} \equiv[\mathbf{I}-(1-f) \mathbf{S}]^{-1} \quad(\text { elastic case })
$$

where $\mathbf{D}$ denotes the overall strain rate tensor, $\mathbf{I}$ the unit fourth-rank tensor and $\mathbf{S}$ Eshelby (1957)'s first tensor, the expressions of the components of which are recalled in Appendix A for completeness. The fourth-rank "strain localization tensor" $\mathbf{L}^{\text {el }}$ here possesses the same symmetries as $\mathbf{S}$, that is, invariance upon interchange of the first two or last two indices, but not upon interchange of the two groups of indices.

In the case of plastic materials, we propose to heuristically replace equation (1) by

$$
\mathbf{D}^{v}=\mathbf{L}: \mathbf{D} \quad \text { (plastic case), }
$$

where the new localization tensor $\mathbf{L}$ is to be obtained from the elastic one $\mathbf{L}^{\mathrm{el}}$ through 
multiplication of some of its components by suitable correction factors. (In more recent works of Ponte-Castaneda and coworkers, see for instance (Agoras and Ponte-Castaneda, 2013), expressions of the void strain rate of type (2) have been proposed but with localization tensors $\mathbf{L}$ not directly related to $\mathbf{L}^{\mathrm{el}}$ and depending in a complex way on the stress state. The present work favors a simpler, "pragmatic" approach).

In the sequel, the directions $x, y$ and $z$ are defined, like in Part I, as parallel to the major, intermediate and minor semi-axes $a, b, c$ of the void.

\subsection{Change of variables}

One difficulty raised by the introduction of plastic corrections into Ponte-Castaneda and Zaidman (1994)'s elastic formula (1) is that not all components of $\mathbf{D}^{v}$ require corrected expressions. For instance, the famous equation resulting from matrix incompressibility

$$
D_{m}^{v}=\frac{D_{m}}{f}
$$

where $D_{m}^{v} \equiv \frac{1}{3} \operatorname{tr} \mathbf{D}^{v}$ denotes the mean strain rate of the void and $D_{m} \equiv \frac{1}{3} \operatorname{tr} \mathbf{D}$ the overall mean strain rate, being exact and independent of the constitutive law, must not be corrected. This is not the only example: in some special cases purely geometrical considerations imply relations independent of the constitutive law between certain components of $\mathbf{D}^{v}$ and $\mathbf{D}$; for instance $D_{x x}^{v}=D_{x x}$ for cylindrical voids.

For this reason, we introduce the following change of variables aimed at distinguishing between components of $\mathbf{D}^{v}$ requiring corrected expressions or not (bearing in mind the case just mentioned of cylindrical voids which necessates to distinguish between the $x x$ components of the tensors $\mathbf{D}^{v}$ and $\mathbf{D}$ and their other components):

$$
\left\{\begin{array}{l}
\bar{D}_{1}^{v} \equiv D_{m}^{v} \\
\bar{D}_{2}^{v} \equiv D_{x x}^{v}-D_{m}^{v} \\
\bar{D}_{3}^{v} \equiv D_{y y}^{v}-D_{z z}^{v} \\
\bar{D}_{4}^{v} \equiv D_{x y}^{v} \\
\bar{D}_{5}^{v} \equiv D_{x z}^{v} \\
\bar{D}_{6}^{v} \equiv D_{y z}^{v}
\end{array} ; \quad\left\{\begin{array}{l}
\bar{D}_{1} \equiv D_{m} \\
\bar{D}_{2} \equiv D_{x x}-D_{m} \\
\bar{D}_{3} \equiv D_{y y}-D_{z z} \\
\bar{D}_{4} \equiv D_{x y} \\
\bar{D}_{5} \equiv D_{x z} \\
\bar{D}_{6} \equiv D_{y z} .
\end{array}\right.\right.
$$

The "elastic" and "plastic" equations (1) and (2) then read in terms of the 6-vectors $\overline{\mathbf{D}}^{v} \equiv\left(\bar{D}_{\alpha}^{v}\right)_{1 \leq \alpha \leq 6}$ and $\overline{\mathbf{D}} \equiv\left(\bar{D}_{\alpha}\right)_{1 \leq \alpha \leq 6}$ :

$$
\overline{\mathbf{D}}^{v}=\overline{\mathbf{L}}^{\mathrm{el}} \cdot \overline{\mathbf{D}} \quad \text { (elastic case) } ; \quad \overline{\mathbf{D}}^{v}=\overline{\mathbf{L}} \cdot \overline{\mathbf{D}} \quad \text { (plastic case) }
$$

where $\overline{\mathbf{L}}^{\mathrm{el}} \equiv\left(\bar{L}_{\alpha \beta}^{\mathrm{el}}\right)_{1 \leq \alpha, \beta \leq 6}$ and $\overline{\mathbf{L}} \equiv\left(\bar{L}_{\alpha \beta}\right)_{1 \leq \alpha, \beta \leq 6}$ are $6 \times 6$ matrices. Because of the orthotropy of the overall behavior in the principal axes of the void, $\overline{\mathbf{L}}^{\mathrm{el}}$ and $\overline{\mathbf{L}}$ are of the 
form (omitting the possible "exponent" el):

$$
\overline{\mathbf{L}}=\left(\begin{array}{cccccc}
\bar{L}_{11} & \bar{L}_{12} & \bar{L}_{13} & 0 & 0 & 0 \\
\bar{L}_{21} & \bar{L}_{22} & \bar{L}_{23} & 0 & 0 & 0 \\
\bar{L}_{31} & \bar{L}_{32} & \bar{L}_{33} & 0 & 0 & 0 \\
0 & 0 & 0 & \bar{L}_{44} & 0 & 0 \\
0 & 0 & 0 & 0 & \bar{L}_{55} & 0 \\
0 & 0 & 0 & 0 & 0 & \bar{L}_{66}
\end{array}\right)
$$

and their components are related to those of the fourth-rank tensors $\mathbf{L}^{\mathrm{el}}$ and $\mathbf{L}$ by the following relations resulting from lengthy but straightforward calculations:

$$
\begin{aligned}
& \left\{\begin{array}{l}
\bar{L}_{11}=1 / f \quad ; \quad \bar{L}_{12}=\bar{L}_{13}=0 \\
\left\{\begin{array}{l}
\bar{L}_{21}=L_{x x x x}+L_{x x y y}+L_{x x z z}-1 / f \\
\bar{L}_{22}=\left(2 L_{x x x x}-L_{x x y y}-L_{x x z z}\right) / 2 \\
\bar{L}_{23}=\left(L_{x x y y}-L_{x x z z}\right) / 2 \\
\bar{L}_{31}=L_{y y x x}+L_{y y y y}+L_{y y z z}-L_{z z x x}-L_{z z y y}-L_{z z z z} \\
\bar{L}_{32}=\left(2 L_{y y x x}-L_{y y y y}-L_{y y z z}-2 L_{z z x x}+L_{z z y y}+L_{z z z z}\right) / 2 \\
\bar{L}_{33}=\left(L_{y y y y}-L_{y y z z}-L_{z z y y}+L_{z z z z}\right) / 2
\end{array}\right. \\
\bar{L}_{44}=2 L_{x y x y} \quad ; \quad \bar{L}_{55}=2 L_{x z x z} \quad ; \quad \bar{L}_{66}=2 L_{y z y z}
\end{array}\right. \\
& \Leftrightarrow\left\{\begin{array}{l}
\left\{\begin{array}{l}
L_{x x x x}=\left(\bar{L}_{21}+2 \bar{L}_{22}+1 / f\right) / 3 \\
L_{x x y y}=\left(\bar{L}_{21}-\bar{L}_{22}+3 \bar{L}_{23}+1 / f\right) / 3 \\
L_{x x z z}=\left(\bar{L}_{21}-\bar{L}_{22}-3 \bar{L}_{23}+1 / f\right) / 3 \\
L_{y y x x}=\left(-\bar{L}_{21}-2 \bar{L}_{22}+\bar{L}_{31}+2 \bar{L}_{32}+2 / f\right) / 6 \\
L_{y y y y}=\left(-\bar{L}_{21}+\bar{L}_{22}-3 \bar{L}_{23}+\bar{L}_{31}-\bar{L}_{32}+3 \bar{L}_{33}+2 / f\right) / 6 \\
L_{y y z z}=\left(-\bar{L}_{21}+\bar{L}_{22}+3 \bar{L}_{23}+\bar{L}_{31}-\bar{L}_{32}-3 \bar{L}_{33}+2 / f\right) / 6 \\
L_{z z x x}=\left(-\bar{L}_{21}-2 \bar{L}_{22}-\bar{L}_{31}-2 \bar{L}_{32}+2 / f\right) / 6 \\
L_{z z y y}=\left(-\bar{L}_{21}+\bar{L}_{22}-3 \bar{L}_{23}-\bar{L}_{31}+\bar{L}_{32}-3 \bar{L}_{33}+2 / f\right) / 6 \\
L_{z z z z}=\left(-\bar{L}_{21}+\bar{L}_{22}+3 \bar{L}_{23}-\bar{L}_{31}+\bar{L}_{32}+3 \bar{L}_{33}+2 / f\right) / 6
\end{array}\right. \\
L_{x y x y}=\bar{L}_{44} / 2 \quad ; \quad L_{x z x z}=\bar{L}_{55} / 2 \quad ; \quad L_{y z y z}=\bar{L}_{66} / 2
\end{array}\right.
\end{aligned}
$$

where account has been taken of equation (3). 


\subsection{Definition of correction factors}

The components of the matrix $\overline{\mathbf{L}}$ are heuristically assumed to be related to those of the elastic matrix $\overline{\mathbf{L}}^{\mathrm{el}}$ through the relations

$$
\bar{L}_{\alpha \beta} \equiv h_{\alpha \beta} \bar{L}_{\alpha \beta}^{\mathrm{el}} \quad(\text { no sum on } \alpha \text { or } \beta)
$$

where the $h_{\alpha \beta}$ are correction factors accounting for plastic effects. (Note that relations (9) are not of "tensorial nature", that is, they hold only in the specific basis chosen to express the matrices). The matrix $\mathbf{h} \equiv\left(h_{\alpha \beta}\right)_{1 \leq \alpha, \beta \leq 6}$ of these factors may harmlessly be taken in the same form (6) as $\overline{\mathbf{L}}$ and $\overline{\mathbf{L}}^{\mathrm{el}}$, that is with $h_{\alpha \beta}=0$ whenever $\bar{L}_{\alpha \beta}=\bar{L}_{\alpha \beta}^{\mathrm{el}}=0$.

The full numerical determination of the matrix $\mathbf{h}$, for all possible geometries and loadings, is an impossible task. We shall therefore content ourselves with adoption of an interpolation formula expressing it, for a general void, as a linear combination of its values $\mathbf{h}^{\mathrm{sph}}$, $\mathbf{h}^{\text {cyl }}, \mathbf{h}^{\text {sand }}$ for special voids having the shape of a sphere, a circular cylinder and an infinite planar empty layer ("sandwich" case) respectively:

$$
\mathbf{h}=\lambda \mathbf{h}^{\mathrm{sph}}+\mu \mathbf{h}^{\mathrm{cyl}}+\nu \mathbf{h}^{\mathrm{sand}}
$$

where $\lambda, \mu, \nu$ denote "interpolation coefficients" satisfying the conditions $\lambda \geq 0, \mu \geq 0$, $\nu \geq 0, \lambda+\mu+\nu=1$ and depending on the void shape. Note that the interpolation coefficients used in equation (10) are the same for all components of the matrix $\mathbf{h}$.

The matrices $\mathbf{h}^{\mathrm{sph}}$ and $\mathbf{h}^{\text {cyl }}$ applicable to spherical and circular cylindrical voids will be determined numerically. Such a task will however not be necessary for the matrix $\mathbf{h}^{\text {sand }}$ pertaining to the sandwich case. Indeed in such a case the kinematics of the cell consists, whatever the loading, of a combination of rigid-body motions and uniform deformations of the layers surrounding the void, and is independent of the constitutive law. Hence the elastic equation (5) $)_{1}$ applies without any correction to the plastic case, which leads to

$$
\mathbf{h}^{\text {sand }}=\left(\begin{array}{llllll}
1 & 1 & 1 & 0 & 0 & 0 \\
1 & 1 & 1 & 0 & 0 & 0 \\
1 & 1 & 1 & 0 & 0 & 0 \\
0 & 0 & 0 & 1 & 0 & 0 \\
0 & 0 & 0 & 0 & 1 & 0 \\
0 & 0 & 0 & 0 & 0 & 1
\end{array}\right) .
$$

\subsection{Restrictions on correction factors}

The numerical task to follow will be simplified to some extent by some restrictions applying to some components of the matrix $\mathbf{h}$. 
- The first line of $\mathbf{h}$ brings corrections in the first component of the vectorial elastic equation $(5)_{1}$ giving $\bar{D}_{1}^{v}$. But this first component only expresses equation (3), which is exact and independent of the constitutive law. Therefore no correction is needed here, which leads to

$$
h_{11}=h_{12}=h_{13}=1 .
$$

- Consider now the first column of $\mathbf{h}$ and more specifically the coefficients $h_{21}$ and $h_{31}$, since $h_{11}$ is already known. In the spherical case and for an elastic material, symmetry considerations imply that $D_{m}$ can influence neither $D_{x x}^{v}-D_{m}^{v}$ nor $D_{y y}^{v}-D_{z z}^{v}$; hence $\bar{L}_{21}^{\mathrm{el}}$ and $\bar{L}_{31}^{\mathrm{el}}$ are zero and insensitive to corrections, which permits to take $h_{21}^{\mathrm{sph}}=h_{31}^{\mathrm{sph}}=1$. For a circular cylindrical void (of axis parallel to the direction $x$ ), the second component of the vectorial elastic equation $(5)_{1}$ giving $\bar{D}_{2}^{v}$ is a consequence of the relation $D_{x x}^{v}=$ $D_{x x}$ plus equation (3) which are both exact and independent of the constitutive law, so it does not require any correction, implying that $h_{21}^{\text {cyl }}=1$; and in elasticity symmetry considerations imply that $D_{m}$ cannot influence $D_{y y}^{v}-D_{z z}^{v}$, so that $\bar{L}_{31}^{\mathrm{el}}$ is zero and insensitive to corrections, thus permitting to take $h_{31}^{\text {cyl }}=1$. Finally, in the sandwich case we have seen that $h_{21}^{\text {sand }}=h_{31}^{\text {sand }}=1$ also. The coefficients $h_{21}$ and $h_{31}$ are then unity in all three "reference cases" used in the interpolation formula (10) which therefore leads to

$$
h_{21}=h_{31}=1
$$

in the general case.

Equations (12) and (13) lead to a matrix $\mathbf{h}$ of the form

$$
\mathbf{h}=\left(\begin{array}{cccccc}
1 & 1 & 1 & 0 & 0 & 0 \\
1 & h_{22} & h_{23} & 0 & 0 & 0 \\
1 & h_{32} & h_{33} & 0 & 0 & 0 \\
0 & 0 & 0 & h_{44} & 0 & 0 \\
0 & 0 & 0 & 0 & h_{55} & 0 \\
0 & 0 & 0 & 0 & 0 & h_{66}
\end{array}\right)
$$

which reduces the numerical task to the determination of the seven coefficients $h_{22}, h_{23}$, $h_{32}, h_{33}, h_{44}, h_{55}$ and $h_{66}$.

- The change of variables (4) distinguishes the $x x$ components of the tensors $\mathbf{D}^{v}, \mathbf{D}$ from their other components. In the general ellipsoidal case this does not raise any problem since the direction $x$ is unambiguously defined as parallel to the major semi-axis $a$ of the void. In the special case of an oblate spheroidal void, however, the direction $x$ may be chosen arbitrarily within the plane containing the two major semi-axes, so that the question arises whether equation $(5)_{2}$ is invariant with respect to the choice made, that is whether it respects transverse isotropy with respect to the direction $z$.

In the spherical case, symmetry considerations imply that the components $\bar{L}_{22}^{\mathrm{el}}, \bar{L}_{33}^{\mathrm{el}}$, $\bar{L}_{44}^{\mathrm{el}}, \bar{L}_{55}^{\mathrm{el}}, \bar{L}_{66}^{\mathrm{el}}$ are all equal and must undergo identical corrections: $h_{22}^{\mathrm{sph}}=h_{33}^{\mathrm{sph}}=h_{44}^{\mathrm{sph}}=$ $h_{55}^{\mathrm{sph}}=h_{66}^{\mathrm{sph}} \equiv h^{\mathrm{sph}}$. Also, $\bar{L}_{23}^{\mathrm{el}}=\bar{L}_{32}^{\mathrm{el}}=0$, which permits to freely choose $h_{23}^{\mathrm{sph}}$ and $h_{32}^{\mathrm{sph}}$. Provided that the choice $h_{23}^{\mathrm{sph}}=h_{32}^{\mathrm{sph}}=h^{\mathrm{sph}}$ is made, the coefficients $h_{22}, h_{23}, h_{32}, h_{33}$, $h_{44}, h_{55}, h_{66}$ are all equal for a spherical void like for a sandwich, and therefore also for 
an oblate spheroidal void considered in the present approach as intermediate between the two (equation (10) with $\mu=0$ ): $h_{22}^{\text {obl }}=h_{23}^{\text {obl }}=h_{32}^{\text {obl }}=h_{33}^{\text {obl }}=h_{44}^{\text {obl }}=h_{55}^{\text {obl }}=h_{66}^{\text {obl }} \equiv h^{\text {obl }}$.

As a consequence, equations (5) read in the oblate spheroidal case:

$$
\left\{\begin{array}{lll}
\bar{D}_{1}^{v}=\bar{D}_{1} / f ; \bar{D}_{\alpha}^{v}=\bar{L}_{\alpha 1}^{\mathrm{el}} \bar{D}_{1}+\sum_{\beta=2}^{6} \bar{L}_{\alpha \beta}^{\mathrm{el}} \bar{D}_{\beta} & \text { for } \alpha=2, \ldots, 6 & \text { (elastic case) } \\
\bar{D}_{1}^{v}=\bar{D}_{1} / f ; \bar{D}_{\alpha}^{v}=\bar{L}_{\alpha 1}^{\mathrm{el}} \bar{D}_{1}+\sum_{\beta=2}^{6} h^{\mathrm{obl}} \bar{L}_{\alpha \beta}^{\mathrm{el}} \bar{D}_{\beta} & \text { for } \alpha=2, \ldots, 6 & \text { (plastic case) }
\end{array}\right.
$$

or equivalently, in symbolic form,

$$
\left(D_{m}^{v}, \mathbf{D}^{v \prime}\right)=\boldsymbol{\Phi}^{\mathrm{el}}\left(D_{m}, \mathbf{D}^{\prime}\right) \quad(\text { elastic case }) ;\left(D_{m}^{v}, \mathbf{D}^{v \prime}\right)=\boldsymbol{\Phi}^{\mathrm{el}}\left(D_{m}, h^{\mathrm{obl}} \mathbf{D}^{\prime}\right) \quad \text { (plastic case) }
$$

where $\mathbf{D}^{v \prime} \equiv \mathbf{D}^{v}-D_{m}^{v} \mathbf{1}$ and $\mathbf{D}^{\prime} \equiv \mathbf{D}-D_{m} \mathbf{1}$ denote the deviators of $\mathbf{D}^{v}$ and $\mathbf{D}$. Thus in the plastic case $\left(D_{m}^{v}, \mathbf{D}^{v^{\prime}}\right)$ is obtained from $\left(D_{m}, \mathbf{D}^{\prime}\right)$ by simply transforming this vector into $\left(D_{m}, h^{\mathrm{obl}} \mathbf{D}^{\prime}\right)$ prior to applying the "elastic operator" $\boldsymbol{\Phi}^{\mathrm{el}}$. Since both operations respect transverse isotropy with respect to the direction $z$, their composition respects it.

The conclusion is that the choice $h_{23}^{\mathrm{sph}}=h_{32}^{\mathrm{sph}}=h^{\mathrm{sph}}$ permits to respect the necessary transverse isotropy of the proposed expression $(5)_{2}$ of $\mathbf{D}^{v}$ with respect to the direction $z$ in the oblate spheroidal case.

\section{Expression of the strain rate of ellipsoidal voids - Numerical determination of plastic corrections}

\subsection{Calculation of the strain and rotation rates of the void}

'The reader is referred to Section 2 of Part I for a general presentation of the numerical procedure employed, including the numerical evaluation of the macroscopic stresses. In this part, the strain and rotation tensors of the void are also needed, so an explanation of the numerical calculation of these tensors is in order.

For ellipsoidal geometries, the components of the strain tensor $\mathbf{E}^{v}$ of the void are calculated from the formula

$$
E_{i j}^{v}=\frac{1}{\operatorname{vol}(\omega)} \int_{\partial \omega} \frac{1}{2}\left(u_{i} n_{j}+u_{j} n_{i}\right) d S
$$

where $\omega$ denotes the void, $\partial \omega$ its boundary and $\mathbf{n}$ the unit outward normal vector to this boundary. (There is no need to calculate the rotation tensor of the void which is automatically zero since for such geometries, we consider only overall strain tensors $\mathbf{E}$ having the same principal directions as the void).

For cylindrical geometries, the components of $\mathbf{E}^{v}$ are obtained in the same way and those of the rotation tensor $\chi^{v}$ of the void from the formula

$$
\chi_{i j}^{v}=\frac{1}{\operatorname{vol}(\omega)} \int_{\partial \omega} \frac{1}{2}\left(u_{i} n_{j}-u_{j} n_{i}\right) d S .
$$

In this case the numerical calculation of the integrals $\int_{\partial \omega} u_{i} n_{j} d S$ in equations (16) and 
(17) may seem problematic since neither the top surface of the void $(\partial \omega)^{\mathrm{T}}$ nor its bottom one $(\partial \omega)^{\mathrm{B}}$ are meshed. But since $\mathbf{n}=\mathbf{e}_{x}$ and $-\mathbf{e}_{x}$ on $(\partial \omega)^{\mathrm{T}}$ and $(\partial \omega)^{\mathrm{B}}$ respectively, the integrals over $(\partial \omega)^{\mathrm{T}}$ and $(\partial \omega)^{\mathrm{B}}$ need only be calculated for $j=x$, and their sum then reduces to $\int_{(\partial \omega)^{B}} \Delta u_{i} d S$, where the $\Delta u_{i}$ are given by equations $(5)_{2,3,4}$ of Part I; the calculation is thus straightforward.

In the sequel, the strain and rotation tensors $\mathbf{E}^{v}, \boldsymbol{\chi}^{v}$ of the void thus obtained numerically are identified, up to some unimportant positive multiplicative constant, to the theoretical strain rate and rotation rate tensors $\mathbf{D}^{v}, \boldsymbol{\Omega}^{v}$ of this void (because the displacement field $\mathbf{u}$ of the finite element simulations may be identified to the theoretical velocity field $\mathbf{v}$ of limit-analysis, see Appendix A of Part I).

\subsection{Spherical case}

It has already been noted in Subsection 2.4 that in the spherical case, the matrix $\mathbf{h} \equiv \mathbf{h}^{\text {sph }}$ must be of the form

$$
\mathbf{h}^{\mathrm{sph}}=\left(\begin{array}{cccccc}
1 & 1 & 1 & 0 & 0 & 0 \\
1 & h^{\mathrm{sph}} & h^{\mathrm{sph}} & 0 & 0 & 0 \\
1 & h^{\mathrm{sph}} & h^{\mathrm{sph}} & 0 & 0 & 0 \\
0 & 0 & 0 & h^{\mathrm{sph}} & 0 & 0 \\
0 & 0 & 0 & 0 & h^{\mathrm{sph}} & 0 \\
0 & 0 & 0 & 0 & 0 & h^{\mathrm{sph}}
\end{array}\right),
$$

which reduces the task to determination of a single coefficient $h^{\text {sph }}$, which may depend only upon the porosity $f$ and the macroscopic stress tensor $\boldsymbol{\Sigma}$. (This conclusion holds only within the framework of approximations made; the full rigorous determination of the strain rate of a spherical void goes beyond the determination of a single coefficient correcting the elastic law (1), as will be clear below).

Quite remarkably, a complete numerical study of the coefficient $h^{\text {sph }}$ as a function of the stress state is possible. Indeed the complete isotropy of the spherical geometry permits to choose the directions $x, y, z$ parallel to the principal directions of the stress tensor $\Sigma$ corresponding to its principal values $\Sigma_{I} \geq \Sigma_{I I} \geq \Sigma_{I I I}$ (in this order). The tensor $\Sigma$ then reduces to three independent components only, $\Sigma_{x x}=\Sigma_{I}, \Sigma_{y y}=\Sigma_{I I}, \Sigma_{z z}=\Sigma_{I I I}$. The overall yield locus being thus a mere 2D surface in a $3 \mathrm{D}$ space may be parametrized by the triaxiality $T$ and the Lode parameter $\omega$ or angle $\phi$ defined by

$$
T \equiv \frac{\Sigma_{m}}{\Sigma_{e q}} \quad ; \quad \omega \equiv \cos (3 \phi) \equiv \frac{27}{2} \operatorname{det} \frac{\Sigma^{\prime}}{\Sigma_{e q}} \quad\left(0^{\circ} \leq \phi \leq 60^{\circ}\right)
$$

where $\Sigma_{m} \equiv \frac{1}{3} \operatorname{tr} \boldsymbol{\Sigma}$ denotes the mean stress, $\boldsymbol{\Sigma}^{\prime} \equiv \boldsymbol{\Sigma}-\Sigma_{m} \mathbf{1}$ the stress deviator and 
$\Sigma_{e q} \equiv\left(\frac{3}{2} \Sigma^{\prime}: \Sigma^{\prime}\right)^{1 / 2}$ the von Mises equivalent stress; $\Sigma_{I}, \Sigma_{I I}, \Sigma_{I I I}$ are then given by

$$
\left\{\begin{array}{l}
\Sigma_{I} / \Sigma_{e q}=T+\frac{2}{3} \cos \phi \\
\Sigma_{I I} / \Sigma_{e q}=T+\frac{2}{3} \cos (\phi-2 \pi / 3) \\
\Sigma_{I I I} / \Sigma_{e q}=T+\frac{2}{3} \cos (\phi+2 \pi / 3)
\end{array}\right.
$$

A full exploration of the range of variation of $T$ and $\omega$ or $\phi$ is perfectly feasible, a single calculation being sufficient at each point $\boldsymbol{\Sigma}$ of the yield surface since the direction of the overall strain rate $\mathbf{D}$ is fixed there as normal to this surface.

An important remark must however be made about the assumed relation (9) connecting the components of the elastic and plastic "localization matrices" $\overline{\mathbf{L}}^{\mathrm{el}}, \overline{\mathbf{L}}$. Using the classical expression of Eshelby's first tensor $\mathbf{S}$ for the spherical geometry and equations $(1)_{2}$ and (7), one easily sees that $\bar{L}_{\alpha \beta}^{\mathrm{el}}=\frac{5}{3+2 f} \delta_{\alpha \beta}$ for $2 \leq \alpha, \beta \leq 6$; this means that in elastic materials, the deviators $\mathbf{D}^{v \prime}$ and $\mathbf{D}^{\prime}$ of the strain rates $\mathbf{D}^{v}$ and $\mathbf{D}$ are connected through the relation

$$
\mathbf{D}^{v \prime}=\frac{5}{3+2 f} \mathbf{D}^{\prime} \quad \text { (elastic case) }
$$

and therefore collinear. In plastic materials, the assumed relation (9), combined with equation (18), yields $\bar{L}_{\alpha \beta}=\frac{5}{3+2 f} h^{\mathrm{sph}} \delta_{\alpha \beta}$ for $2 \leq \alpha, \beta \leq 6$; this means that

$$
\mathbf{D}^{v \prime}=\frac{5}{3+2 f} h^{\mathrm{sph}} \mathbf{D}^{\prime} \quad \text { (plastic case) }
$$

which again implies collinearity of $\mathbf{D}^{v \prime}$ and $\mathbf{D}^{\prime}$. Thus the assumed relation (9) makes the implicit hypothesis that for spherical voids, the strain rate deviators $\mathbf{D}^{v \prime}$ and $\mathbf{D}^{\prime}$ remain collinear in plasticity like in elasticity. But this property of collinearity, which is a rigorous consequence of linearity and isotropy in elasticity, has no reason to remain true in plasticity. Thus the assumed relation (9) involves an error $\mathcal{E}$ the importance of which may be appreciated in the spherical case from the lack of collinearity of the strain rate deviators $\mathbf{D}^{v \prime}$ and $\mathbf{D}^{\prime}$.

The numerical study must therefore encompass the determination of both the coefficient $h^{\text {sph }}$ and the error $\mathcal{E}$. To define these quantities within the numerical context, it is necessary to introduce some notations. For any symmetric second-rank tensor $\mathbf{T}$, let $\mathbf{T}_{\mathrm{dg}} \in \mathbb{R}^{3}$ denote the vector made of its diagonal components $T_{x x}, T_{y y}, T_{z z}$ in the orthonormal basis defined by the directions $x, y, z$ introduced above. Note that if $\mathbf{T}$ is traceless, $\mathbf{T}_{\mathrm{dg}}$ lies in the plane $\mathcal{P}$ orthogonal to the vector of components $1,1,1$. Note also that since, for symmetry reasons, the tensors $\mathbf{D}^{v^{\prime}}$ and $\mathbf{D}^{\prime}$ are diagonal in the same basis as the tensor $\boldsymbol{\Sigma}$, they are completely characterized by the vectors $\mathbf{D}_{\mathrm{dg}}^{v^{\prime}}$ and $\mathbf{D}_{\mathrm{dg}}^{\prime}$ of their diagonal components. Now define the following vectors, to be obtained numerically:

$$
\mathbf{U} \equiv \frac{\mathbf{D}^{\prime}{ }_{\mathrm{dg}}}{\left\|\mathbf{D}_{\mathrm{dg}}^{\prime}\right\|} \quad ; \quad \mathbf{U}^{\perp} \equiv \frac{1}{\sqrt{3}}\left(\begin{array}{l}
1 \\
1 \\
1
\end{array}\right) \times \mathbf{U} \quad ; \quad \mathbf{U}^{v} \equiv \frac{3+2 f}{5} \frac{\mathbf{D}_{\mathrm{dg}}^{v^{\prime}}}{\left\|\mathbf{D}_{\mathrm{dg}}^{\prime}\right\|}
$$


where $\left\|\mathbf{D}_{\text {dg }}^{\prime}\right\| \equiv\left(\mathbf{D}_{\mathrm{dg}}^{\prime} \cdot \mathbf{D}_{\mathrm{dg}}^{\prime}\right)^{1 / 2}$ denotes the euclidian norm of the vector $\mathbf{D}_{\mathrm{dg}}^{\prime} ; \mathbf{U} \in \mathcal{P}$ is the unit vector collinear to $\mathbf{D}^{\prime}{ }_{\mathrm{dg}}, \mathbf{U}^{\perp}$ is the unit vector orthogonal to $\mathbf{U}$ in $\mathcal{P}$, and $\mathbf{U}^{v} \in \mathcal{P}$ is a vector collinear to $\mathbf{D}^{v^{\prime}}$,g , normalized in such a way that $\left\|\mathbf{U}^{v}\right\|=1$ in the elastic case (see equation (21)). If the formula (22) resulting from the assumed relation (9) were exact, it would imply $\mathbf{U}^{v}=h^{\mathrm{sph}} \mathbf{U}$ and therefore $h^{\mathrm{sph}}=\mathbf{U}^{v}$.U and $\mathbf{U}^{v} \cdot \mathbf{U}^{\perp}=0$. It is therefore logical to numerically define the coefficient $h^{\text {sph }}$ and the normalized (dimensionless) error $\mathcal{E}$ involved in equation (9) as

$$
h^{\mathrm{sph}} \equiv \mathbf{U}^{v} \cdot \mathbf{U} \quad ; \quad \mathcal{E} \equiv \mathbf{U}^{v} \cdot \mathbf{U}^{\perp}
$$

It is important to note that in these expressions, all vectors are to be taken from the numerical calculations, meaning that here like in the sequel, the numerical results will be exploited (to define the correction coefficients looked for, and here also the error $\mathcal{E}$ ) by using the overall strain rate $\mathbf{D}$ determined numerically, not that resulting from Madou and Leblond (2012a,b)'s theoretical criterion and the associated associated flow rule. This will avoid introducing errors resulting from the approximate nature of this criterion in the relation connecting $\mathbf{D}^{v}$ and $\mathbf{D}$. (Of course, in practical applications, the errors resulting from the criterion and the expression proposed for $\mathbf{D}^{v}$ will add up). It is also necessary to do so to comply with our ambition of defining such a relation independently of the approximate yield criterion used.

A bibliographic digression is finally in order before the presentation of numerical results. A large number of works have studied the deformation of spherical or spheroidal voids in plastic matrices subjected to various loadings by numerical methods. However, in the vast majority of these works, based on the finite element method and initiated by the pioneering study of Koplik and Needleman (1988), the entire deformation history of the void was studied (the geometry was continuously updated), and a limited number of loadings was considered. These works therefore did not provide a comprehensive study, for a broad class of loadings, of the void strain rate for a given, fixed void geometry, which is what is wanted here.

A few studies, however, based on numerical limit-analysis, did investigate the void strain rate for a fixed void geometry and a variety of loadings: for plastic matrices and spherical voids, the work of Rice and Tracey (1969), later completed by Huang (1991); and for viscoplastic matrices, the work of Budiansky et al. (1982) for spherical voids and that of Lee and Mear (1992) for spheroidal ones. Budiansky et al. (1982) were notably the first to note the following typical plastic effect, referred to hereafter as the BHS effect: ${ }^{1}$ a spherical cavity, subjected to some axisymmetric load with major axial stress $\left(\Sigma_{I}>\Sigma_{I I}=\Sigma_{I I I}\right)$, tends to become oblate, instead of prolate as one would intuitively expect, when the triaxiality is high enough. But the matrix was infinite in all these studies, resulting in a zero porosity; detailed comparisons of their results with those presented below would be rather pointless since nonzero porosities are considered here, and the influence of the porosity will be seen to be quite large. (In an extension of Lee and Mear (1992)'s work, Yee and Mear (1996) considered spheroidal voids embedded in a finite viscoplastic Norton matrix, but with values of the Norton exponent limited to 4, too low to adequately

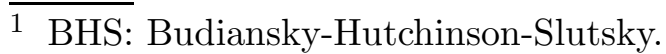


represent the ideal-plastic behavior considered here).

In fact the only works usable here for detailed comparisons seem to be those of Gologanu (1997); Gologanu et al. (1997), who performed numerical limit-analyses of hollow spheres providing the void strain rate for several nonzero porosities and various loadings. Even in these works, however, the loadings considered were all axisymmetric, implying values of the Lode angle $\phi$ limited to $0^{\circ}$ and $60^{\circ}$ and, for symmetry reasons, automatic collinearity of the strain rate deviators $\mathbf{D}^{v \prime}$ and $\mathbf{D}^{\prime}$ (zero error $\mathcal{E}$ ).

We now present numerical results. Figure 1 displays the normalized error $\mathcal{E}$, obtained through numerical limit-analyses (analogous to those of Part I) of a hollow sphere with a typical porosity of 0.01 , as a function of the triaxiality $T$, for various values of the Lode angle $\phi$. One sees that for $\phi=0^{\circ}$ and $60^{\circ}, \mathcal{E}$ is zero $\left(\mathbf{D}^{v \prime}\right.$ and $\mathbf{D}^{\prime}$ are collinear), as expected. For other values of the Lode angle, however, $\mathcal{E}$ is nonzero $\left(\mathbf{D}^{v^{\prime}}\right.$ and $\mathbf{D}^{\prime}$ are not collinear $)$ and increases with $T$; for $\phi=30^{\circ}$ and $T=3$, it amounts to as much as 0.6 , indicating a very significant non-collinearity of $\mathbf{D}^{v \prime}$ and $\mathbf{D}^{\prime}$. (Note that this non-collinearity does not result from an error introduced by the implicit approximation that the void retains a truly ellipsoidal shape when deformed; indeed the "numerical definition" (17) of the rotation tensor of the void is independent of such an approximation).

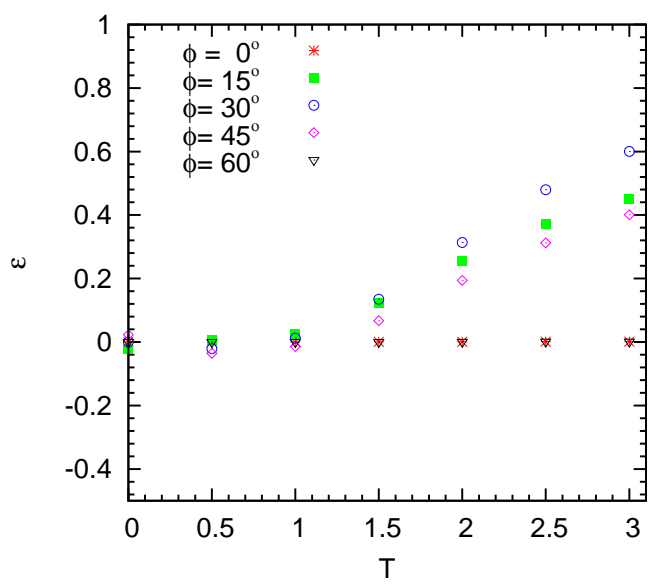

Fig. 1. Normalized error made by equation (9), for a hollow sphere with porosity $f=0.01$

The non-collinearity of $\mathbf{D}^{v \prime}$ and $\mathbf{D}^{\prime}$ appears to have remained unnoticed in previous works. It must be stressed, however, to be small for all types of loadings and triaxialities not exceeding about 1.5, and nil for axisymmetric loadings and all triaxialities. In other words, the only loading cases where it is really important are those close to a state of pure shear with a large superimposed hydrostatic stress.

In the sequel, we shall neglect this non-collinearity and stick to equation (9) in spite of the error involved, which admittedly is not always very small, and whose implications have not been assessed. The reason is that incorporating this non-collinearity into the expression of $\mathbf{D}^{v \prime}$ would be very difficult. The major difficulty does not arise in the spherical case itself; in this case one may perfectly envisage to express the relation connecting $\mathbf{D}^{v \prime}$ and $\mathbf{D}^{\prime}$ in the principal basis of the latter tensor and add a term in the right-hand side of equation (22) breaking the collinearity, adjusting coefficients in this term to match the numerical results of Figure 1. The true difficulty arises in the case of an arbitrary ellipsoidal void; 
for such a void the principal bases of the ellipsoid and the overall strain rate are different, so if a similar additional term were to be introduced in the expression of $\mathbf{D}^{v \prime}$ proposed (equations $(5)_{2}$ and (9)), it is not clear in which basis this term should be expressed, and how one could respect the necessary relations resulting from possible symmetries of the geometry and/or the loading.

Figures 2 and 3 show numerical results (marked "Num") for the coefficient $h^{\text {sph }}$. Figure 2 is for axisymmetric loadings with major axial stress $\left(\phi=0^{\circ}, \Sigma_{I}>\Sigma_{I I}=\Sigma_{I I I}\right)$ or lateral stress $\left(\phi=60^{\circ}, \Sigma_{I}=\Sigma_{I I}>\Sigma_{I I I}\right)$ and two porosities, $f=0.01$ and 0.001 ; the earlier numerical results (marked "Num GLD") of Gologanu (1997) and Gologanu et al. (1997) for such loadings are also provided here. Figure 3 is for non-axisymmetric loadings $\left(\phi \neq 0^{\circ}, \phi \neq 60^{\circ}\right)$ but a single porosity, $f=0.01$. Several observations are in order:

- The new results are in excellent agreement with the earlier ones of Gologanu (1997); Gologanu et al. (1997) in spite of the widely different numerical methods used, which like in Part I is a strong argument in favor of the correctness and accuracy of all of them.

- The coefficient $h^{\mathrm{sph}}$ strongly depends on the triaxiality $T$ for all values of $\phi$, and becomes negative for large $T$, implying in particular that for $\phi=0^{\circ}$ and $60^{\circ}$, the strain rate deviators $\mathbf{D}^{v^{\prime}}$ and $\mathbf{D}^{\prime}$ become negatively collinear, in contradiction with intuition but in agreement with the well-known BHS effect.

- The coefficient $h^{\mathrm{sph}}$ also depends notably on $\phi$, and very strongly on $f$ (compare Figures 2 (a) and 2(c) for instance). The marked influence of the Lode angle upon the void strain rate is in contrast with its very small influence upon the overall criterion, evidenced for instance by Gologanu (1997)'s numerical calculations of the yield loci of hollow spheres subjected to conditions of homogeneous boundary strain rate. (In Madou and Leblond (2012a,b)'s model, the latter influence is neglected since their criterion for spherical voids is identical to Leblond et al. (1994)'s variant of that of Gurson (1977)).

Figures 2 and 3 also compare numerical values of $h^{\mathrm{sph}}$ to those (marked "Model") provided by the following approximate formula:

$$
h^{\mathrm{sph}}=\frac{1}{7}(9-10 \sqrt{f}+8 f)-(1-\sqrt{f})^{8} \frac{11 T^{4}}{5\left(11+|T|^{3}\right)}\left[1+\frac{\omega}{3} \operatorname{sgn}(T)\right]
$$

where $\operatorname{sgn}(T)$ denotes the sign of $T$; in addition, Figure 2 shows the predictions (marked "Model GLD") of earlier formulae of Gologanu et al. (1997) applicable to the sole values $\phi=0^{\circ}$ and $60^{\circ}$. The new formula (25) can be observed to make a good job of reproducing the numerical results in all cases, and bring a definite improvement over the old ones for $\phi=0^{\circ}$ and $60^{\circ}$, at least for $f=0.01$ and high values of $T .{ }^{2}$ In addition, it possesses the following nice theoretical properties:

- The coefficient $h^{\mathrm{sph}}$ remains unchanged upon change of sign of the stress tensor $\boldsymbol{\Sigma}$ (since both $T$ and $\omega$ change sign, see equations (19)), which means that the relation between $\mathbf{D}^{v \prime}$ and $\mathbf{D}^{\prime}$ remains the same. This is necessary since parity of the local (von Mises)

$\overline{2}$ Note however that the large exponent 8 in the term $(1-\sqrt{f})^{8}$ is not determined accurately; the values 7 or 9 would certainly be just as acceptable. The same remark applies to other large exponents appearing in various approximate formulae proposed below. 


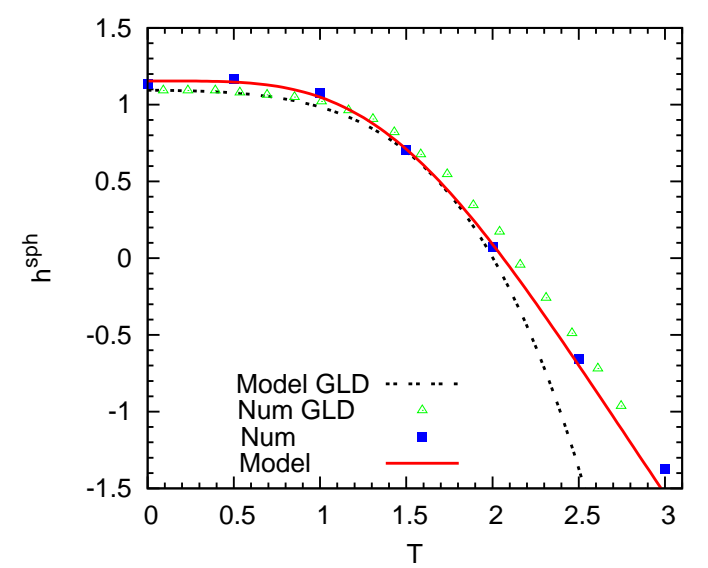

(a) $f=0.01, \phi=0^{\circ}$

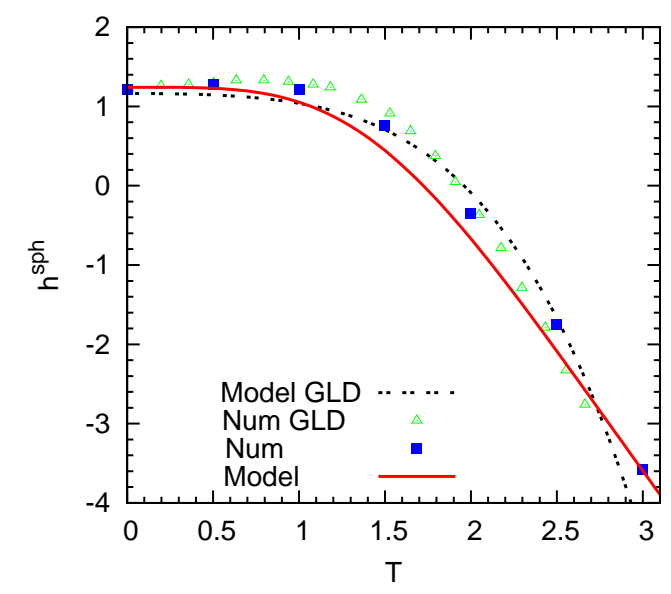

(c) $f=0.001, \phi=0^{\circ}$

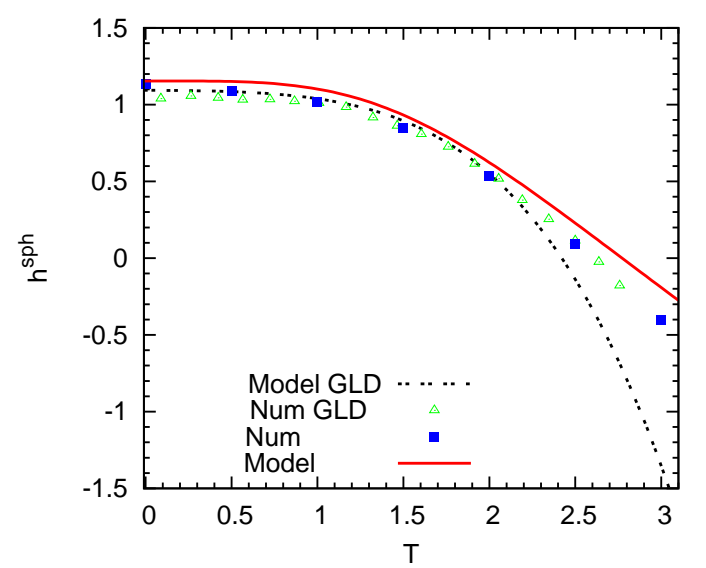

(b) $f=0.01, \phi=60^{\circ}$

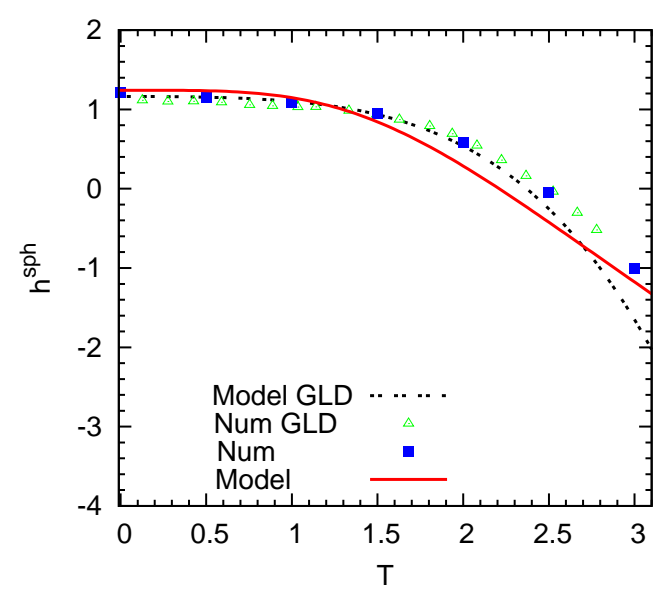

(d) $f=0.001, \phi=60^{\circ}$

Fig. 2. The coefficient $h^{\text {sph }}$ for axisymmetric loadings as a function of $T$, for $f=0.01$ and 0.001

criterion implies that when $\boldsymbol{\Sigma}$ changes sign, the entire velocity field does the same so that both $\mathbf{D}^{v^{\prime}}$ and $\mathbf{D}^{\prime}$ simultaneously change sign.

- The $\sqrt{f}$-dependence of $h^{\mathrm{sph}}$ ensures that this coefficient varies quickly with the porosity when it is small, in agreement with numerical observations.

- For $f=1$, the coefficient $h^{\text {sph }}$ is unity so that equation (22) reduces to $\mathbf{D}^{v \prime}=\mathbf{D}^{\prime}$, as desired in this case since the void then fills the whole representative cell.

\subsection{Circular cylindrical case}

For a circular cylindrical void (of axis parallel to the direction $x$ ), it has already been remarked in Subsection 2.4 that the second component of the elastic equation $(5)_{1}$ giving $\bar{D}_{2}^{v}$, being exact and independent of the constitutive law, requires no correction, implying that $h_{22}^{\text {cyl }}=h_{23}^{\text {cyl }}=1$. Furthermore in elasticity symmetry considerations imply that $D_{x x}-$ $D_{m}$ cannot influence $D_{y y}^{v}-D_{z z}^{v}$, so that $\bar{L}_{32}^{\text {el }}$ is zero and insensitive to corrections, thus permitting to take $h_{32}^{\text {cyl }}=1$. Symmetries also imply that $h_{33}^{\text {cyl }}=h_{66}^{\text {cyl }}$ and $h_{44}^{\text {cyl }}=h_{55}^{\text {cyl }}$. By 


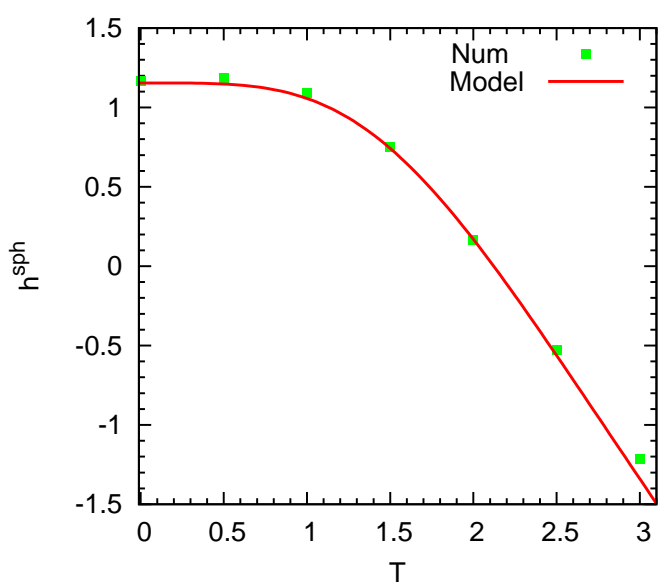

(a) $f=0.01, \phi=15^{\circ}$

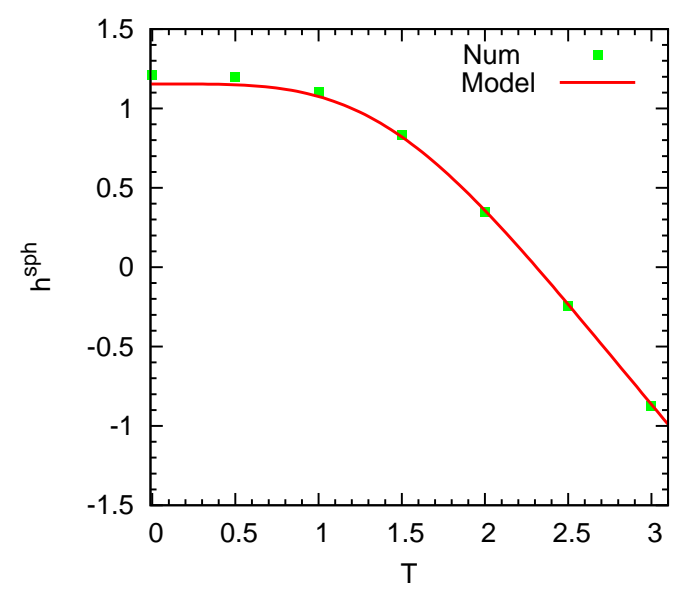

(b) $f=0.01, \phi=30^{\circ}$

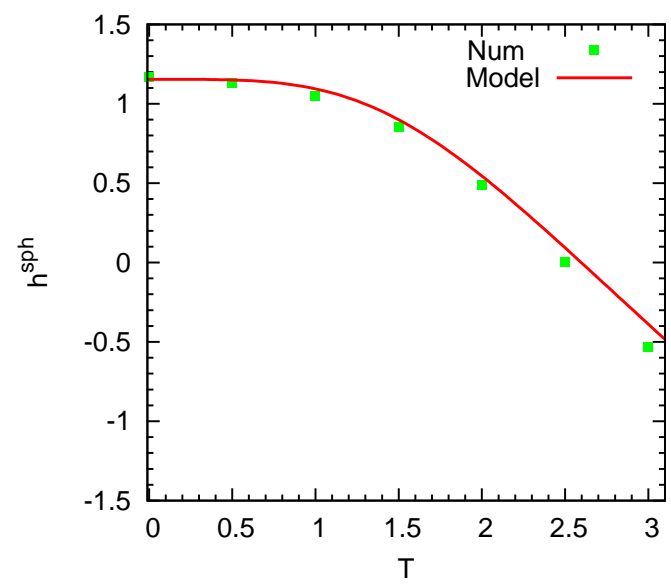

(c) $f=0.01, \phi=45^{\circ}$

Fig. 3. The coefficient $h^{\text {sph }}$ for non-axisymmetric loadings as a function of $T$, for $f=0.01$ equation (14), the matrix $\mathbf{h}^{\text {cyl }}$ is thus of the form

$$
\mathbf{h}^{\mathrm{cyl}}=\left(\begin{array}{cccccc}
1 & 1 & 1 & 0 & 0 & 0 \\
1 & 1 & 1 & 0 & 0 & 0 \\
1 & 1 & h_{33}^{\mathrm{cyl}} & 0 & 0 & 0 \\
0 & 0 & 0 & h_{44}^{\mathrm{cyl}} & 0 & 0 \\
0 & 0 & 0 & 0 & h_{44}^{\mathrm{cyl}} & 0 \\
0 & 0 & 0 & 0 & 0 & h_{33}^{\mathrm{cyl}}
\end{array}\right)
$$

where again the coefficients $h_{33}^{\text {cyl }}$ and $h_{44}^{\text {cyl }}$ may depend only on the porosity $f$ and the macroscopic stress tensor $\boldsymbol{\Sigma}$.

Unlike in the spherical case, however, a full numerical study of these coefficients is impossible, because the lower degree of symmetry of the circular cylindrical geometry makes the variety of loading cases to be considered too great. We shall therefore only study a limited number of "suitably chosen" loading cases, hoping (without definite proof) to thus 
capture essential effects.

Consider the coefficient $h_{33}^{\text {cyl }}$ first. Using the expression of Eshelby's tensor $\mathbf{S}$ for the circular cylindrical geometry and equations $(1)_{2},(7)$ and (9), one sees that the third component of the vectorial equation $(5)_{2}$ reads

$$
D_{y y}^{v}-D_{z z}^{v}=\frac{2}{1+f} h_{33}^{\mathrm{cyl}}\left(D_{y y}-D_{z z}\right) \quad \text { (plastic case). }
$$

Since the quantities $D_{y y}^{v}-D_{z z}^{v}$ and $D_{y y}-D_{z z}$ involve only diagonal components of $\mathbf{D}^{v}$ and

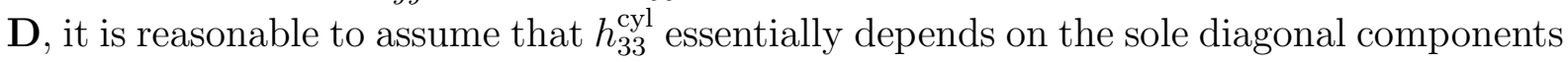
of $\boldsymbol{\Sigma}$, and thus concentrate on macroscopic stresses having the same principal directions as the void. The overall yield locus then becomes a $2 \mathrm{D}$ surface in the $3 \mathrm{D}$ space of principal stresses, and we calculate $h_{33}^{\text {cyl }}$ (using equation (27) with the numerical values of $D_{y y}^{v}, D_{z z}^{v}$, $\left.D_{y y}, D_{z z}\right)$ along the intersections of this surface and three planes of respective equations $\Sigma_{x x}=\left(\Sigma_{y y}+\Sigma_{z z}\right) / 2, \Sigma_{x x}=\Sigma_{y y}$ and $\Sigma_{x x}=0$ (plane stress case). ${ }^{3}$

Figure 4 shows the results obtained for the porosities $f=0.01$ and 0.001 . (The plane stress case is considered only for the former porosity). The coefficient $h_{33}^{\text {cyl }}$ is plotted here versus the lateral triaxiality $T_{h}$ defined by

$$
T_{h} \equiv \frac{\frac{1}{2}\left(\Sigma_{y y}+\Sigma_{z z}\right)}{\Sigma_{e q}}
$$

The results are quite similar for the three types of loadings for $f=0.01$, a little less so for $f=0.001$, suggesting that $h_{33}^{\text {cyl }}$ may be considered to approximately depend on $\boldsymbol{\Sigma}$ via the sole parameter $T_{h}$.

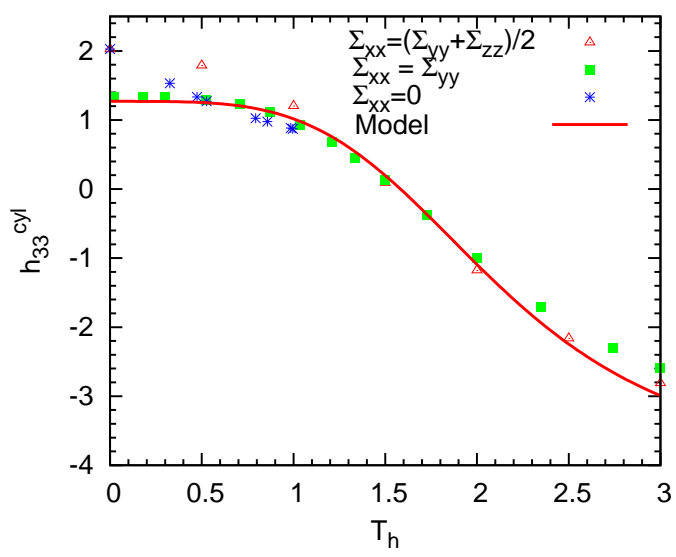

(a) $f=0.01$

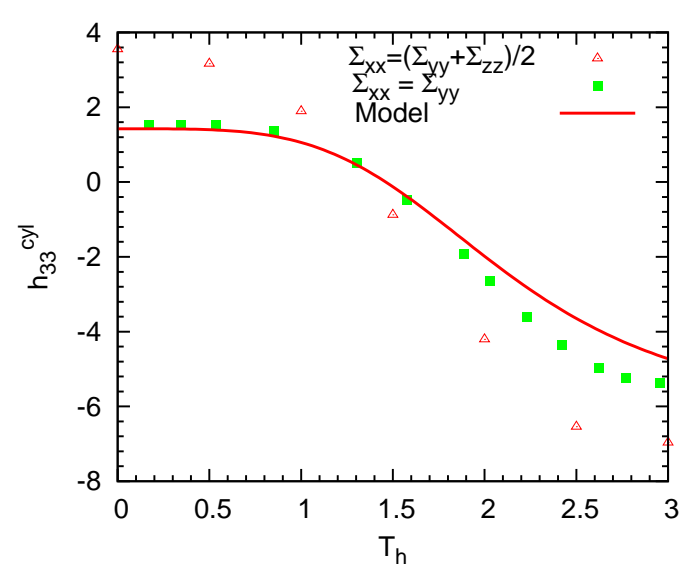

(b) $f=0.001$

Fig. 4. The coefficient $h_{33}^{\text {cyl }}$ as a function of $T_{h}$, for $f=0.01$ and $f=0.001$

It is important to note that the coefficient $h_{33}^{\text {cyl }}$, just like $h^{\text {sph }}$, becomes negative for large values of $T_{h}$. This means that a circular cylindrical void subjected to distinct principal lateral stresses may grow more in the direction of the minor stress than in that of the

$\overline{3}$ The stresses $\Sigma_{y y}$ and $\Sigma_{z z}$ are taken distinct in all cases, otherwise $D_{y y}^{v}-D_{z z}^{v}$ and $D_{y y}-D_{z z}$ would be zero and $h_{33}^{\text {cyl }}$ could not be determined. 
major one, if the lateral triaxiality is high enough. This counterintuitive phenomenon is the exact equivalent, for circular cylindrical voids, of the BHS effect for spherical voids. It was noticed for the first time by Fleck and Hutchinson (1986), whose results thus find a confirmation here.

Figure 4 also shows values of $h_{33}^{\text {cyl }}$ (marked "Model") corresponding to the following approximate formula:

$$
h_{33}^{\mathrm{cyl}} \equiv \frac{1}{2}(3-5 \sqrt{f}+4 f)-(1-\sqrt{f})^{5} \frac{9 T_{h}^{4}}{20+T_{h}^{4}}
$$

which may be observed to reproduce the numerical results with a reasonable accuracy. This formula also possesses nice theoretical features analogous to those of formula (25) for the coefficient $h^{\text {sph }}$, see Subsection 3.2.

Consider now the coefficient $h_{44}^{\text {cyl }}$. Using again the expression of Eshelby's tensor $\mathbf{S}$ for the circular cylindrical geometry and equations $(1)_{2},(7)$ and (9), one gets for the fourth component of the vectorial equation $(5)_{2}$ :

$$
D_{x y}^{v}=\frac{2}{1+f} h_{44}^{\mathrm{cyl}} D_{x y} \quad(\text { plastic case })
$$

This formula makes it clear that $h_{44}^{\text {cyl }}$ cannot be determined by considering only stress tensors $\boldsymbol{\Sigma}$ having the same principal directions as the void. In order to however limit the variety of possible loading cases, we consider only tensors $\boldsymbol{\Sigma}$ corresponding to the superposition of an axisymmetric load $\left(\Sigma_{x x} \neq \Sigma_{y y}=\Sigma_{z z}\right)$ and a shear stress $\Sigma_{x y}$. The trace of the yield locus in the $3 \mathrm{D}$ space of such tensors is again a $2 \mathrm{D}$ surface, and we calculate $h_{44}^{\text {cyl }}$ (using equation (30) with the numerical values of $D_{x y}^{v}$ and $D_{x y}$ ) along the intersections of this surface and four planes of respective equations $\Sigma_{y y} / \Sigma_{x x}=1 / 2,3 / 4$, 1 and 2.

Figure 5 shows the results obtained for the porosities $f=0.01$ and 0.001 . The coefficient $h_{44}^{\text {cyl }}$ is plotted as a function of the deviatoric transverse invariant

$$
X \equiv \frac{\Sigma_{x x}-\frac{1}{2}\left(\Sigma_{y y}+\Sigma_{z z}\right)}{\Sigma_{e q}}
$$

(which lies in the interval $[-1,1]$ but may be considered as positive since $h_{44}^{\text {cyl }}$ is invariant in the transformation $\Sigma \rightarrow-\Sigma$ ). Figure 5 clearly shows that $h_{44}^{\text {cyl }}$ also depends to some extent on the ratio $\Sigma_{y y} / \Sigma_{x x}$, especially for $f=0.001$, but this extra dependence is disregarded in the following approximate formula:

$$
h_{44}^{\mathrm{cyl}}=1+\frac{11}{2}(1-\sqrt{f})^{11}-(1-\sqrt{f})^{11} \frac{84 X^{2}}{1+20 X^{2}}
$$

which provides a reasonable "average" representation of the numerical results. 


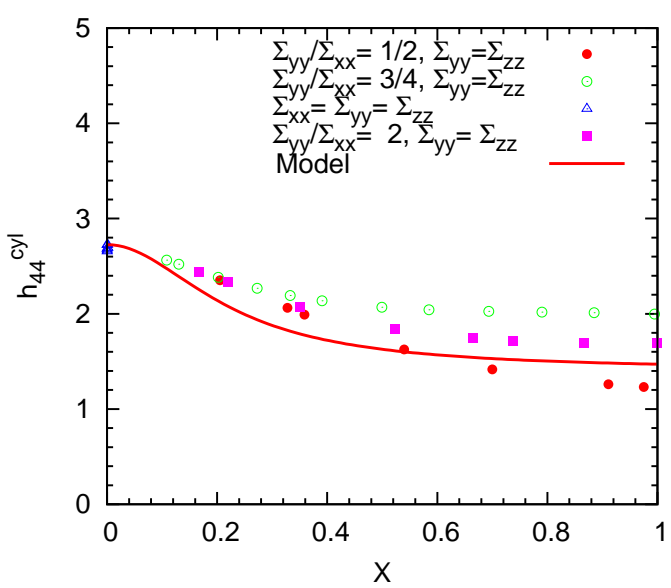

(a) $f=0.01$

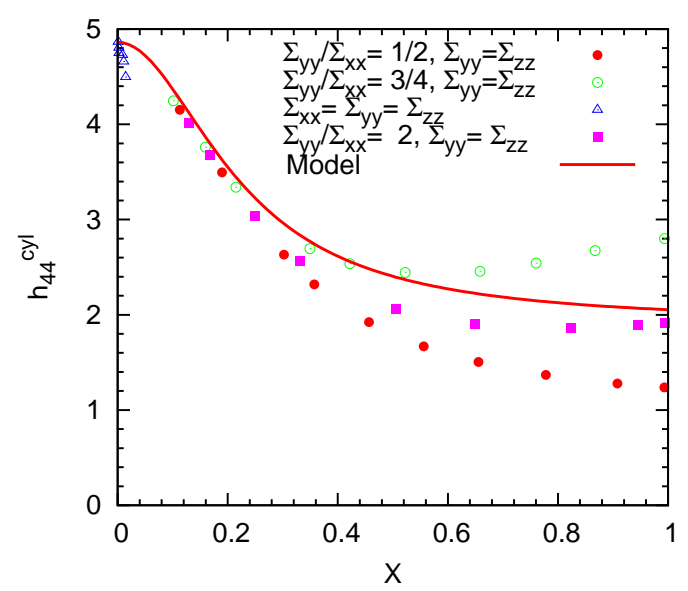

(b) $f=0.001$

Fig. 5. The coefficient $h_{44}^{\text {cyl }}$ as a function of $X$, for $f=0.01$ and $f=0.001$

\subsection{Prolate spheroidal case}

We now consider a prolate spheroidal void (of direction of rotational symmetry $x$ ) of semi-axes $(a, c, c)$. We regard this void as intermediary between a spherical one of semiaxes $(c, c, c)$ and a circular cylindrical one of semi-axes $(+\infty, c, c)$. The expression of the corresponding matrix $\mathbf{h} \equiv \mathbf{h}^{\text {prol }}$ is thus looked for in the form of a linear interpolation between the "extremal values" $\mathbf{h}^{\mathrm{sph}}$ and $\mathbf{h}^{\text {cyl }}$. One possible (but clearly non-unique) choice for the interpolation coefficient to be used is some power of the void eccentricity in the $x z$ plane,

$$
e_{x z} \equiv \frac{\sqrt{a^{2}-c^{2}}}{a}
$$

which lies in the interval $[0,1]$ and takes the values 0 and 1 in the spherical and circular cylindrical cases, respectively. The expression of $\mathbf{h}^{\text {prol }}$ is thus taken in the form

$$
\mathbf{h}^{\mathrm{prol}}\left(f, e_{x z} ; \boldsymbol{\Sigma}\right)=\left(1-e_{x z}^{m}\right) \mathbf{h}^{\mathrm{sph}}(f ; \boldsymbol{\Sigma})+e_{x z}^{m} \mathbf{h}^{\mathrm{cyl}}(f ; \boldsymbol{\Sigma})
$$

for some exponent $m$ to be determined. Temporary explicit indications of the arguments of the functions have been introduce here to underline the choice made of spherical and circular cylindrical voids with the same porosity (and of course subjected to the same stress state) to define the extremal values $\mathbf{h}^{\text {sph }}$ and $\mathbf{h}^{\text {cyl }}$.

By equations (18) and (26), the matrix $\mathbf{h}^{\text {prol }}$ then takes the form

$$
\mathbf{h}^{\mathrm{prol}}=\left(\begin{array}{cccccc}
1 & 1 & 1 & 0 & 0 & 0 \\
1 & h_{22}^{\mathrm{prol}} & h_{22}^{\mathrm{prol}} & 0 & 0 & 0 \\
1 & h_{22}^{\mathrm{prol}} & h_{33}^{\mathrm{prol}} & 0 & 0 & 0 \\
0 & 0 & 0 & h_{44}^{\text {prol }} & 0 & 0 \\
0 & 0 & 0 & 0 & h_{44}^{\text {prol }} & 0 \\
0 & 0 & 0 & 0 & 0 & h_{33}^{\text {prol }}
\end{array}\right)
$$


where

$$
\left\{\begin{array}{l}
h_{22}^{\mathrm{prol}}\left(f, e_{x z} ; \boldsymbol{\Sigma}\right)=\left(1-e_{x z}^{m}\right) h^{\mathrm{sph}}(f ; \boldsymbol{\Sigma})+e_{x z}^{m} \\
h_{33}^{\mathrm{prol}}\left(f, e_{x z} ; \boldsymbol{\Sigma}\right)=\left(1-e_{x z}^{m}\right) h^{\mathrm{sph}}(f ; \boldsymbol{\Sigma})+e_{x z}^{m} h_{33}^{\mathrm{cyl}}(f ; \boldsymbol{\Sigma}) \\
h_{44}^{\mathrm{prol}}\left(f, e_{x z} ; \boldsymbol{\Sigma}\right)=\left(1-e_{x z}^{m}\right) h^{\mathrm{sph}}(f ; \boldsymbol{\Sigma})+e_{x z}^{m} h_{44}^{\mathrm{cyl}}(f ; \boldsymbol{\Sigma}),
\end{array}\right.
$$

$h^{\mathrm{sph}}, h_{33}^{\text {cyl }}$ and $h_{44}^{\text {cyl }}$ being themselves given by equations (25), (29) and (32).

In order to determine the best possible value of the exponent $m$, numerical calculations are performed for a prolate spheroidal void having $(a, b, c)=(5,1,1)$ and $f=0.01$. For simplicity, we envisage only stress tensors $\boldsymbol{\Sigma}$ with the same principal directions as the void and two equal principal stresses; such tensors have $\Sigma_{y y}=\Sigma_{z z} \neq \Sigma_{x x}$ (axisymmetric loadings) or $\Sigma_{x x}=\Sigma_{y y} \neq \Sigma_{z z}$ (non-axisymmetric loadings), and $\phi=0^{\circ}$ or $60^{\circ}$. (The case $\left(\Sigma_{x x}=\Sigma_{z z} \neq \Sigma_{y y}\right)$ need not be considered since it is identical to the case $\left(\Sigma_{x x}=\Sigma_{y y} \neq\right.$ $\left.\left.\Sigma_{z z}\right)\right)$.

Figures 6 and 7 display the results obtained for the components of the deviatoric void strain rate, normalized by the euclidian norm $\|\mathbf{D}\| \equiv(\mathbf{D}: \mathbf{D})^{1 / 2}$ of the tensor $\mathbf{D}$, as a function of the triaxiality $T$; Figure 6 thus shows $\left(D_{x x}^{v}-D_{m}^{v}\right) /\|\mathbf{D}\|$ for axisymmetric stress states and Figure $7,\left(D_{x x}^{v}-D_{m}^{v}\right) /\|\mathbf{D}\|$ and $\left(D_{y y}^{v}-D_{z z}^{v}\right) /\|\mathbf{D}\|$ for non-axisymmetric ones. These figures also display values of these quantities obtained from the theoretical equations $(5)_{2},(9),(35)$ and (36) with the exponent

$$
m=30,
$$

using the numerical values of $D_{x x}, D_{y y}, D_{z z} \cdot{ }^{4}$ The agreement of numerical and theoretical values of $\left(D_{x x}^{v}-D_{m}^{v}\right) /\|\mathbf{D}\|$ and $\left(D_{y y}^{v}-D_{z z}^{v}\right) /\|\mathbf{D}\|$ is quite good for this value.

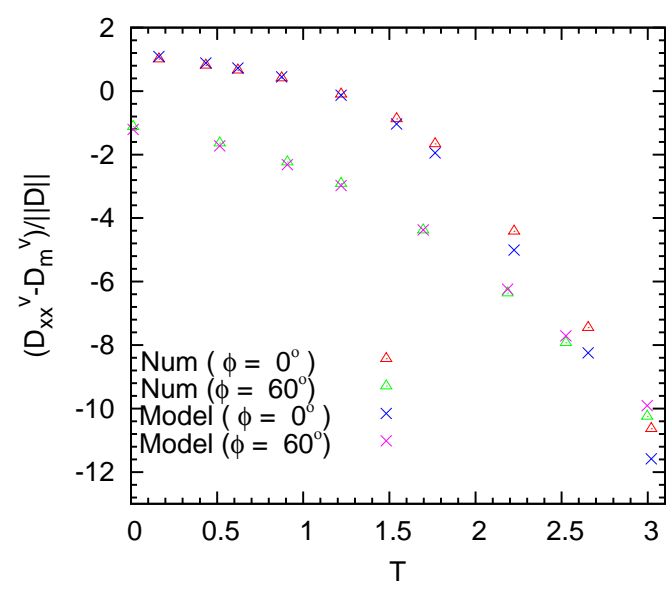

Fig. 6. Normalized void strain rate $\left(D_{x x}^{v}-D_{m}^{v}\right) /\|\mathbf{D}\|$ for a prolate spheroidal void having $(a, b, c)=(5,1,1)$ and $f=0.01$, subjected to axisymmetric loadings $\left(\Sigma_{y y}=\Sigma_{z z} \neq \Sigma_{x x}\right)$

Incidentally, since the value of $m$ given by equation (37) is high, one may think that except for extremely elongated voids, $e_{x z}^{m} \ll 1$ and therefore $\mathbf{h}^{\text {prol }} \simeq \mathbf{h}^{\text {sph }}$. This impression is wrong because not only for extremely long voids but also for moderately elongated ones,

$\overline{4}$ The use of such numerical values, known only discretely, explains why "theoretical" predictions are represented here by discrete points instead of continuous lines. 


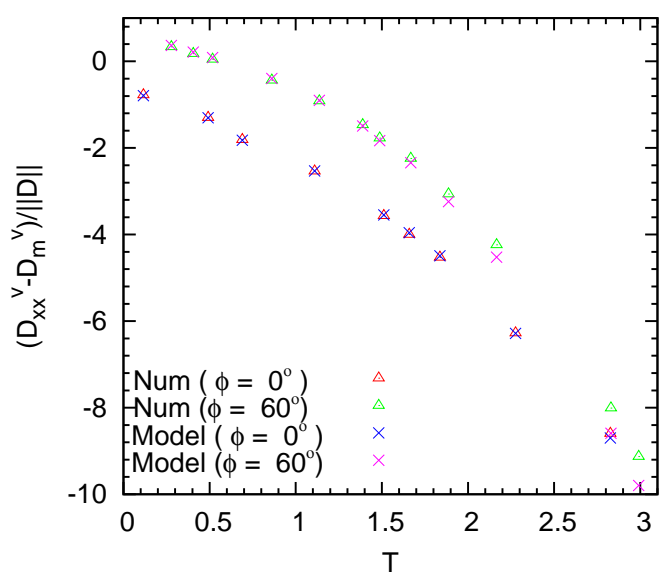

(a) $\left(D_{x x}^{v}-D_{m}^{v}\right) /\|\mathbf{D}\|$

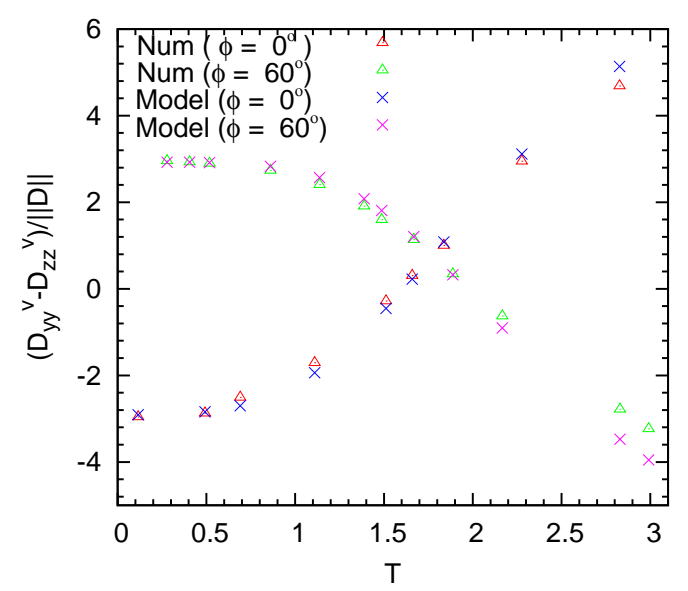

(b) $\left(D_{y y}^{v}-D_{z z}^{v}\right) /\|\mathbf{D}\|$

Fig. 7. Normalized void strain rates $\left(D_{x x}^{v}-D_{m}^{v}\right) /\|\mathbf{D}\|$ and $\left(D_{y y}^{v}-D_{z z}^{v}\right) /\|\mathbf{D}\|$ for a prolate spheroidal void having $(a, b, c)=(5,1,1)$ and $f=0.01$, subjected to non-axisymmetric loadings $\left(\Sigma_{x x}=\Sigma_{y y} \neq \Sigma_{z z}\right)$

$e_{x z}$ is close to 1 and thus $e_{x z}^{m}$ not much smaller than 1 . For the void of moderate aspect ratio $a / c=5$ envisaged in Figures 6 and 7 , for instance, $e_{x z} \simeq 0.980$ and $e_{x z}^{30} \simeq 0.542$, so that $\mathbf{h}^{\text {prol }}$ is about half the sum of $\mathbf{h}^{\text {sph }}$ and $\mathbf{h}^{\text {cyl }}$ and differs a lot from $\mathbf{h}^{\text {sph }}$.

One may also note that the sole consideration, in the numerical simulations just discussed, of tensors $\boldsymbol{\Sigma}$ diagonal in the principal basis of the void, permits to assess the quality of approximation (36) for the coefficients $h_{22}^{\text {prol }}$ and $h_{33}^{\text {prol }}$, but not $h_{44}^{\text {prol }}$ since both $D_{x y}^{v}$ and $D_{x y}$ are zero. Testing this approximation for $h_{44}^{\text {prol }}$ would require considering non-diagonal tensors $\boldsymbol{\Sigma}$, which would necessitate meshing more than $1 / 8$ of the spheroidal cell and result in extremely heavy simulations.

\subsection{Oblate spheroidal case}

We now consider an oblate spheroidal void (of direction of rotational symmetry $z$ ) of semi-axes $(a, a, c)$. We regard this void as intermediary between a spherical one of semiaxes $(c, c, c)$ and a planar layer of semi-axes $(+\infty,+\infty, c)$ (sandwich case), and again use some power of the void eccentricity $e_{x z}$ in the $x z$ plane to interpolate the matrix $\mathbf{h} \equiv \mathbf{h}^{\text {obl }}$ between the extremal values $\mathbf{h}^{\text {sph }}$ and $\mathbf{h}^{\text {sand }}$ at constant porosity:

$$
\mathbf{h}^{\mathrm{obl}}\left(f, e_{x z} ; \boldsymbol{\Sigma}\right)=\left(1-e_{x z}^{n}\right) \mathbf{h}^{\mathrm{sph}}(f ; \boldsymbol{\Sigma})+e_{x z}^{n} \mathbf{h}^{\mathrm{sand}}(f ; \boldsymbol{\Sigma})
$$


where $n$ is an exponent to be determined. By equations (11) and (18), the matrix $\mathbf{h}^{\text {obl }}$ is then of the form

$$
\mathbf{h}^{\mathrm{obl}}=\left(\begin{array}{cccccc}
1 & 1 & 1 & 0 & 0 & 0 \\
1 & h^{\mathrm{obl}} & h^{\mathrm{obl}} & 0 & 0 & 0 \\
1 & h^{\mathrm{obl}} & h^{\mathrm{obl}} & 0 & 0 & 0 \\
0 & 0 & 0 & h^{\mathrm{obl}} & 0 & 0 \\
0 & 0 & 0 & 0 & h^{\mathrm{obl}} & 0 \\
0 & 0 & 0 & 0 & 0 & h^{\mathrm{obl}}
\end{array}\right)
$$

where

$$
h^{\mathrm{obl}}\left(f, e_{x z} ; \boldsymbol{\Sigma}\right)=\left(1-e_{x z}^{n}\right) h^{\mathrm{sph}}(f ; \boldsymbol{\Sigma})+e_{x z}^{n},
$$

$h^{\text {sph }}$ being itself given by equation (25).

Again, we perform numerical calculations for an oblate spheroidal void having $(a, b, c)=$ $(5,5,1)$ and $f=0.01$; we consider only stress tensors $\boldsymbol{\Sigma}$ with the same principal directions as the void and two equal principal stresses, having thus $\Sigma_{x x}=\Sigma_{y y} \neq \Sigma_{z z}$ (axisymmetric loadings) or $\Sigma_{x x}=\Sigma_{z z} \neq \Sigma_{y y}$ (non-axisymmetric loadings), and $\phi=0^{\circ}$ or $60^{\circ}$. Figure 8 shows the computed values of $\left(D_{x x}^{v}-D_{m}^{v}\right) /\|\mathbf{D}\|$ for axisymmetric stress states and Figure 9 those of $\left(D_{x x}^{v}-D_{m}^{v}\right) /\|\mathbf{D}\|$ and $\left(D_{y y}^{v}-D_{z z}^{v}\right) /\|\mathbf{D}\|$ for non-axisymmetric ones, together with those obtained from the theoretical equations $(5)_{2},(9),(39)$ and (40) with the exponent

$$
n=50,
$$

using the numerical values of $D_{x x}, D_{y y}, D_{z z}$. Again, the theoretical formulae reproduce the numerical results with a reasonable accuracy.

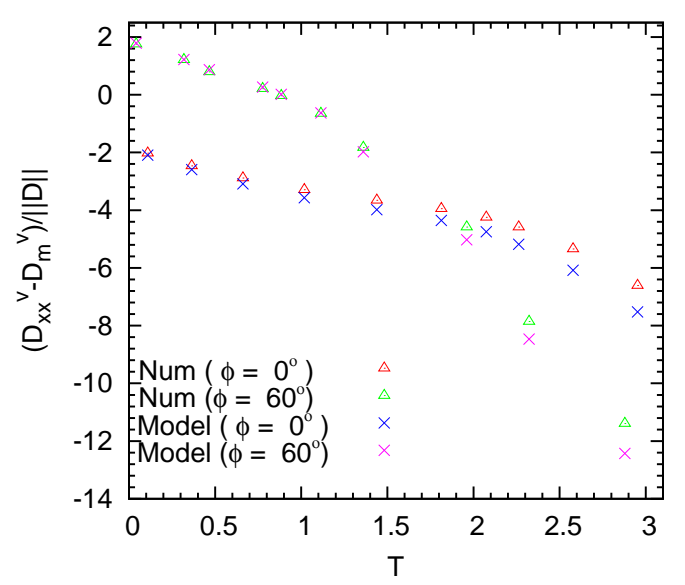

Fig. 8. Normalized void strain rate $\left(D_{x x}^{v}-D_{m}^{v}\right) /\|\mathbf{D}\|$ for an oblate spheroidal void having $(a, b, c)=(5,5,1)$ and $f=0.01$, subjected to axisymmetric loadings $\left(\Sigma_{x x}=\Sigma_{y y} \neq \Sigma_{z z}\right)$

\subsection{General ellipsoidal case}

We finally consider a general ellipsoidal void of semi-axes $(a, b, c)$, and regard it as intermediary between prolate and oblate spheroidal ones of semi-axes $(a, c, c)$ and $(a, a, c)$ 


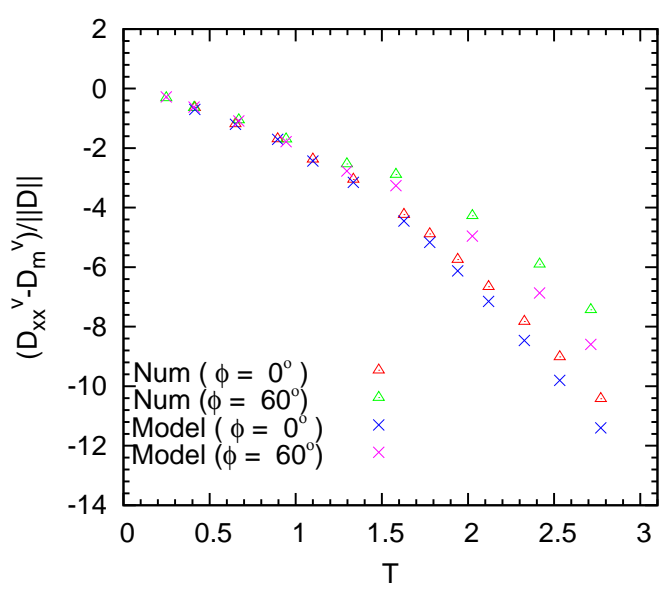

(a) $\left(D_{x x}^{v}-D_{m}^{v}\right) /\|\mathbf{D}\|$

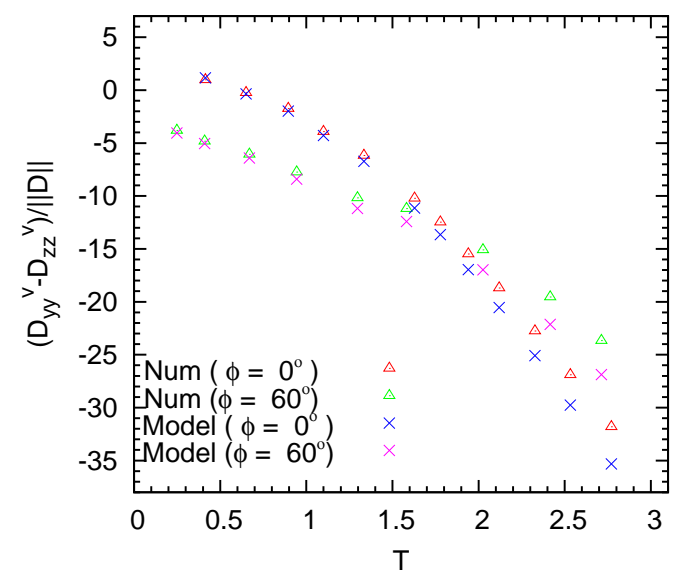

(b) $\left(D_{y y}^{v}-D_{z z}^{v}\right) /\|\mathbf{D}\|$

Fig. 9. Normalized void strain rates $\left(D_{x x}^{v}-D_{m}^{v}\right) /\|\mathbf{D}\|$ and $\left(D_{y y}^{v}-D_{z z}^{v}\right) /\|\mathbf{D}\|$ for an oblate spheroidal void having $(a, b, c)=(5,5,1)$ and $f=0.01$, subjected to non-axisymmetric loadings $\left(\Sigma_{x x}=\Sigma_{z z} \neq \Sigma_{y y}\right)$

respectively, having thus the same eccentricity $e_{x z}$ in the $x z$ plane. To interpolate the matrix $\mathbf{h}$ between the extremal values $\mathbf{h}^{\text {prol }}$ and $\mathbf{h}^{\text {obl }}$ corresponding to these voids, one may use Madou and Leblond (2012a,b)'s parameter

$$
k \equiv \sqrt{\frac{b^{2}-c^{2}}{a^{2}-c^{2}}}
$$

which lies in the interval $[0,1]$ and takes the values 0 and 1 for prolate and oblate spheroidal voids, respectively. Performing again the interpolation at constant porosity, we thus take

$$
\mathbf{h}\left(f, k, e_{x z} ; \boldsymbol{\Sigma}\right)=(1-k) \mathbf{h}^{\mathrm{prol}}\left(f, e_{x z} ; \boldsymbol{\Sigma}\right)+k \mathbf{h}^{\mathrm{obl}}\left(f, e_{x z} ; \boldsymbol{\Sigma}\right) .
$$

The matrix $\mathbf{h}$ is then, by equations (35) and (39), of the form

$$
\mathbf{h}=\left(\begin{array}{cccccc}
1 & 1 & 1 & 0 & 0 & 0 \\
1 & h_{22} & h_{22} & 0 & 0 & 0 \\
1 & h_{22} & h_{33} & 0 & 0 & 0 \\
0 & 0 & 0 & h_{44} & 0 & 0 \\
0 & 0 & 0 & 0 & h_{44} & 0 \\
0 & 0 & 0 & 0 & 0 & h_{33}
\end{array}\right)
$$

where

$$
\left\{\begin{array}{l}
h_{22}\left(f, k, e_{x z} ; \boldsymbol{\Sigma}\right)=(1-k) h_{22}^{\mathrm{prol}}\left(f, e_{x z} ; \boldsymbol{\Sigma}\right)+k h^{\mathrm{obl}}\left(f, e_{x z} ; \boldsymbol{\Sigma}\right) \\
h_{33}\left(f, k, e_{x z} ; \boldsymbol{\Sigma}\right)=(1-k) h_{33}^{\mathrm{prol}}\left(f, e_{x z} ; \boldsymbol{\Sigma}\right)+k h^{\mathrm{obl}}\left(f, e_{x z} ; \boldsymbol{\Sigma}\right) \\
h_{44}\left(f, k, e_{x z} ; \boldsymbol{\Sigma}\right)=(1-k) h_{44}^{\mathrm{prol}}\left(f, e_{x z} ; \boldsymbol{\Sigma}\right)+k h^{\mathrm{obl}}\left(f, e_{x z} ; \boldsymbol{\Sigma}\right),
\end{array}\right.
$$

$h_{22}^{\text {prol }}, h_{33}^{\text {prol }}, h_{44}^{\text {prol }}$ and $h^{\text {obl }}$ being themselves given by equations (36) and (40). 
To assess the accuracy of these approximate formulae, numerical calculations are performed for two general ellipsoidal voids having $(a, b, c)=(10,5,1)$ and $(10,2,1)$ respectively, and $f=0.01$. The stress tensors $\boldsymbol{\Sigma}$ envisaged have the same principal directions as the void and two equal principal stresses, defining thus three types of loadings: $\Sigma_{x x}=\Sigma_{y y} \neq \Sigma_{z z}, \Sigma_{x x}=\Sigma_{z z} \neq \Sigma_{y y}$ and $\Sigma_{y y}=\Sigma_{z z} \neq \Sigma_{x x}$, with the two possibilities $\phi=0^{\circ}$ and $60^{\circ}$ in each case. Figures 10, 11 and 12 shows the computed values of $\left(D_{x x}^{v}-D_{m}^{v}\right) /\|\mathbf{D}\|$ and $\left(D_{y y}^{v}-D_{z z}^{v}\right) /\|\mathbf{D}\|$ for the first void subjected to the three types of loadings, and Figures 13, 14 and 15 do the same for the second one; the predictions of the theoretical equations $(5)_{2},(9),(44)$ and (45), used with the numerical values of $D_{x x}$, $D_{y y}$ and $D_{z z}$, are also shown. The theoretical equations provide a good representation of the numerical results, especially for the first void.

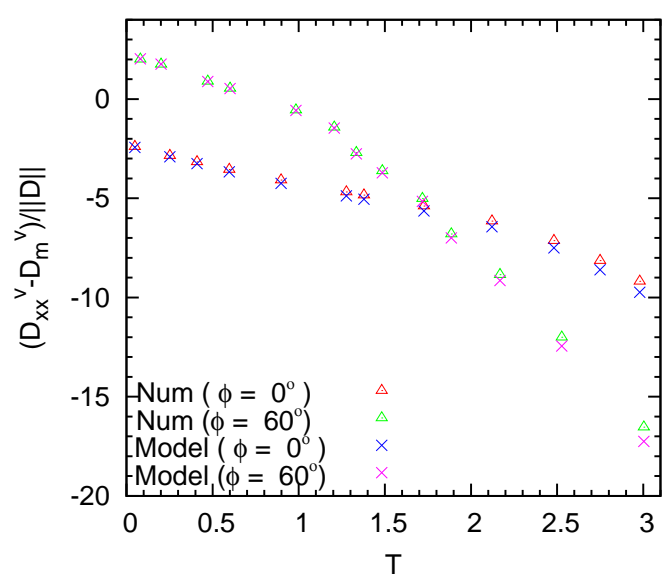

(a) $\left(D_{x x}^{v}-D_{m}^{v}\right) /\|\mathbf{D}\|$

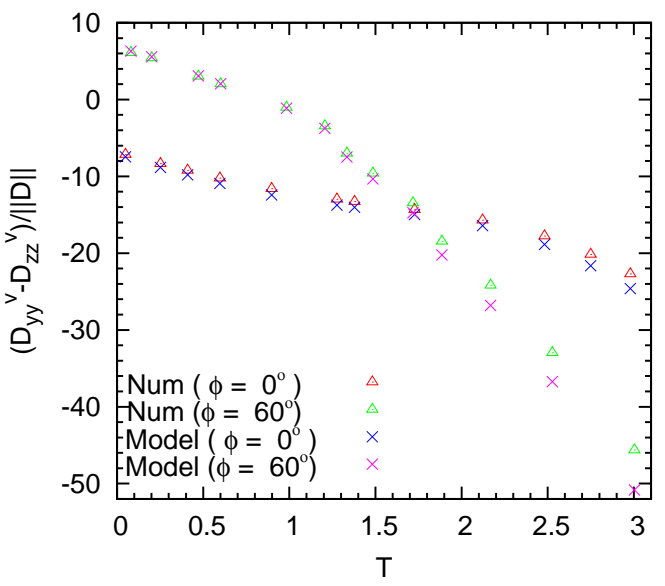

(b) $\left(D_{y y}^{v}-D_{z z}^{v}\right) /\|\mathbf{D}\|$

Fig. 10. Normalized void strain rates $\left(D_{x x}^{v}-D_{m}^{v}\right) /\|\mathbf{D}\|$ and $\left(D_{y y}^{v}-D_{z z}^{v}\right) /\|\mathbf{D}\|$ for an ellipsoidal void having $(a, b, c)=(10,5,1)$ and $f=0.01$, subjected to loadings having $\Sigma_{x x}=\Sigma_{y y} \neq \Sigma_{z z}$

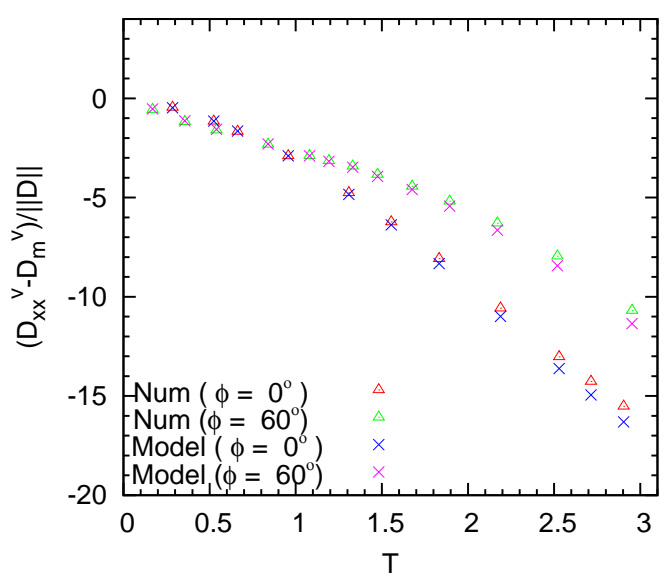

(a) $\left(D_{x x}^{v}-D_{m}^{v}\right) /\|\mathbf{D}\|$

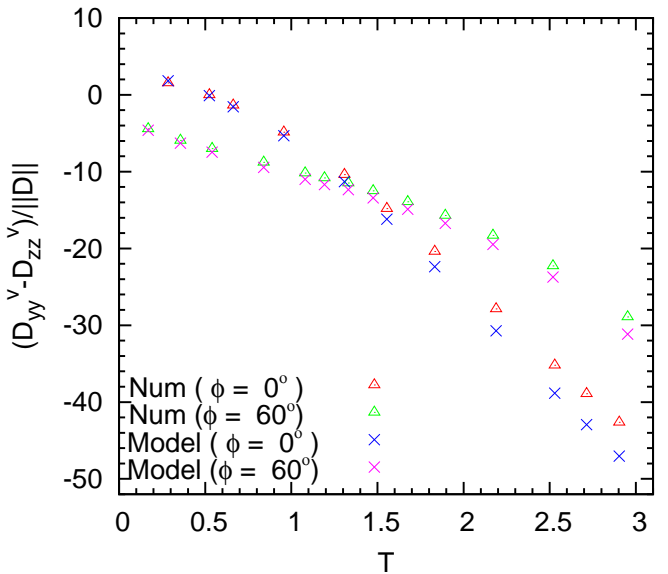

(b) $\left(D_{y y}^{v}-D_{z z}^{v}\right) /\|\mathbf{D}\|$

Fig. 11. Normalized void strain rates $\left(D_{x x}^{v}-D_{m}^{v}\right) /\|\mathbf{D}\|$ and $\left(D_{y y}^{v}-D_{z z}^{v}\right) /\|\mathbf{D}\|$ for an ellipsoidal void having $(a, b, c)=(10,5,1)$ and $f=0.01$, subjected to loadings having $\Sigma_{x x}=\Sigma_{z z} \neq \Sigma_{y y}$ 


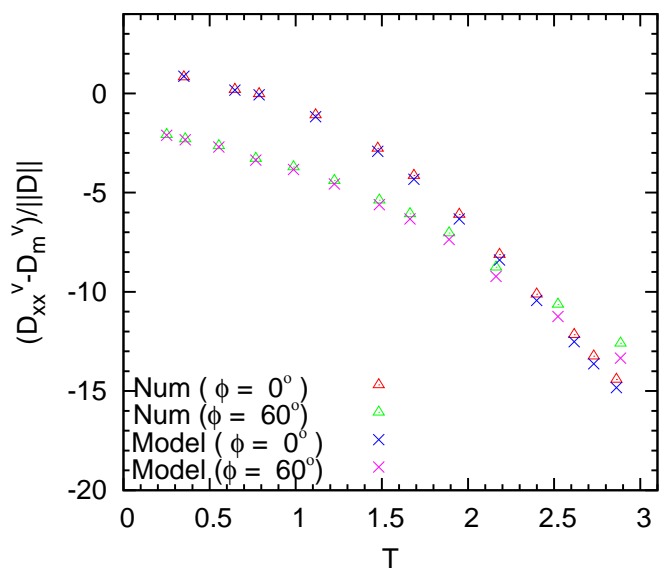

(a) $\left(D_{x x}^{v}-D_{m}^{v}\right) /\|\mathbf{D}\|$

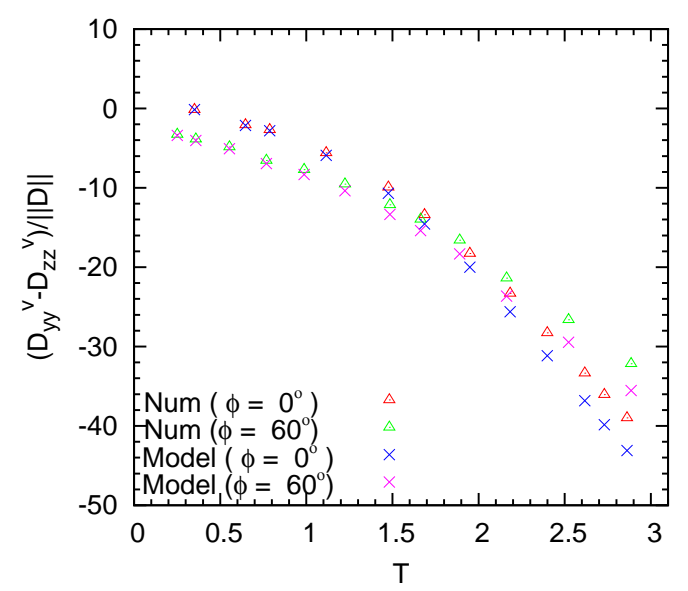

(b) $\left(D_{y y}^{v}-D_{z z}^{v}\right) /\|\mathbf{D}\|$

Fig. 12. Normalized void strain rates $\left(D_{x x}^{v}-D_{m}^{v}\right) /\|\mathbf{D}\|$ and $\left(D_{y y}^{v}-D_{z z}^{v}\right) /\|\mathbf{D}\|$ for an ellipsoidal void having $(a, b, c)=(10,5,1)$ and $f=0.01$, subjected to loadings having $\Sigma_{y y}=\Sigma_{z z} \neq \Sigma_{x x}$

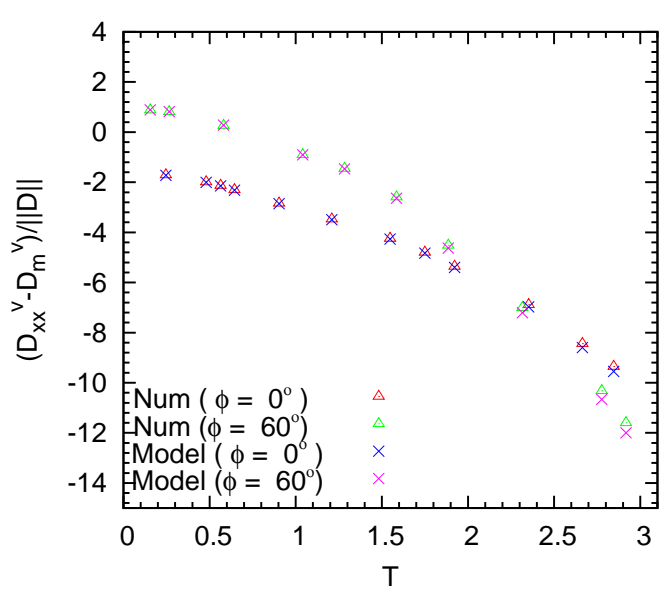

(a) $\left(D_{x x}^{v}-D_{m}^{v}\right) /\|\mathbf{D}\|$

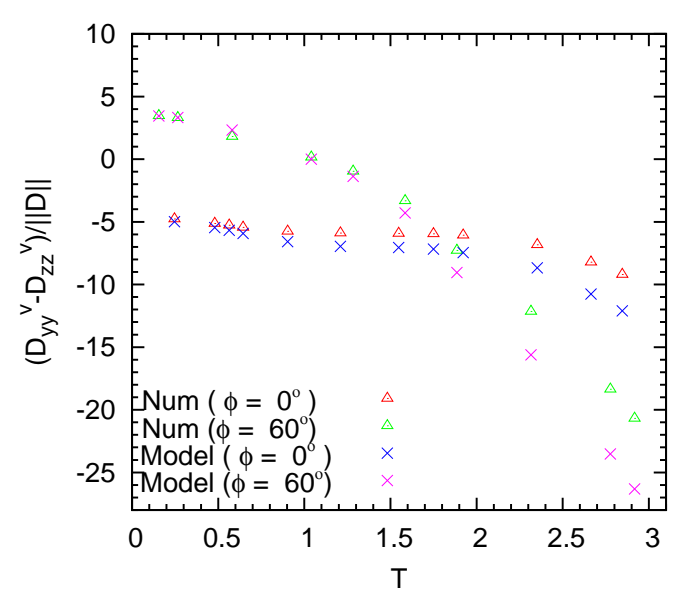

(b) $\left(D_{y y}^{v}-D_{z z}^{v}\right) /\|\mathbf{D}\|$

Fig. 13. Normalized void strain rates $\left(D_{x x}^{v}-D_{m}^{v}\right) /\|\mathbf{D}\|$ and $\left(D_{y y}^{v}-D_{z z}^{v}\right) /\|\mathbf{D}\|$ for an ellipsoidal void having $(a, b, c)=(10,2,1)$ and $f=0.01$, subjected to loadings having $\Sigma_{x x}=\Sigma_{y y} \neq \Sigma_{z z}$

\section{Expression of the rotation rate of ellipsoidal voids}

\subsection{Generalities}

Kailasam and Ponte-Castaneda (1998)'s formula, derived from homogenization theory, for the rotation rate (second-rank antisymmetric tensor) $\Omega^{v}$ of an ellipsoidal void embedded in an isotropic incompressible elastic body, reads

$$
\boldsymbol{\Omega}^{v}=\boldsymbol{\Omega}+\mathbf{R}^{\mathrm{el}}: \mathbf{D} \quad, \quad \mathbf{R}^{\mathrm{el}} \equiv(1-f) \boldsymbol{\Pi}: \mathbf{L}^{\mathrm{el}} \quad(\text { elastic case })
$$

where $\boldsymbol{\Omega}$ denotes the overall rotation rate tensor and $\boldsymbol{\Pi}$ Eshelby (1957)'s second tensor, the expressions of the components of which are recalled in Appendix A. The new fourthrank "rotation localization tensor" $\mathbf{R}^{\mathrm{el}}$ here is, just like $\boldsymbol{\Pi}$, antisymmetric in its first 


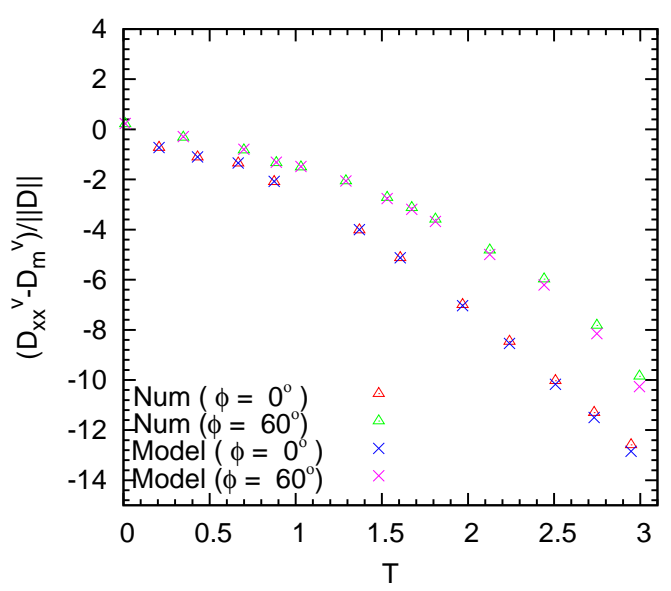

(a) $\left(D_{x x}^{v}-D_{m}^{v}\right) /\|\mathbf{D}\|$

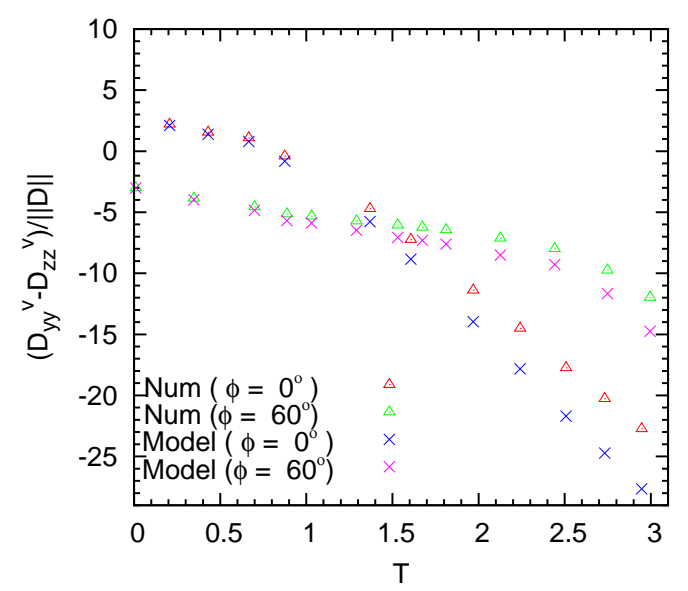

(b) $\left(D_{y y}^{v}-D_{z z}^{v}\right) /\|\mathbf{D}\|$

Fig. 14. Normalized void strain rates $\left(D_{x x}^{v}-D_{m}^{v}\right) /\|\mathbf{D}\|$ and $\left(D_{y y}^{v}-D_{z z}^{v}\right) /\|\mathbf{D}\|$ for an ellipsoidal void having $(a, b, c)=(10,2,1)$ and $f=0.01$, subjected to loadings having $\Sigma_{x x}=\Sigma_{z z} \neq \Sigma_{y y}$

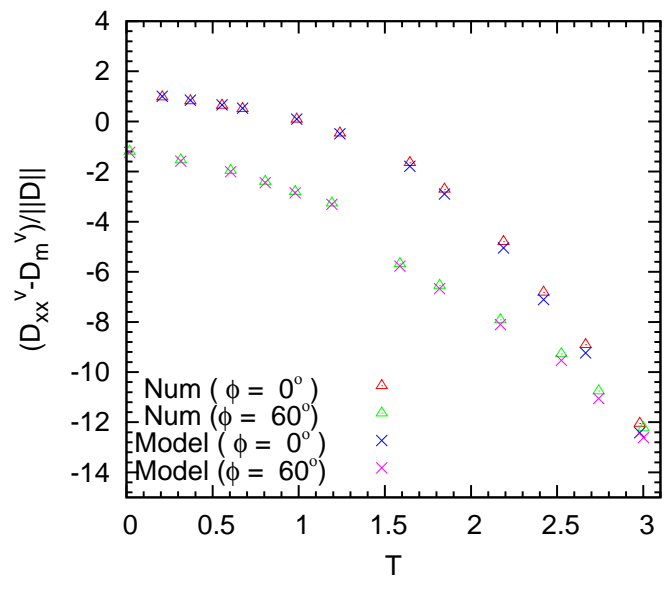

(a) $\left(D_{x x}^{v}-D_{m}^{v}\right) /\|\mathbf{D}\|$

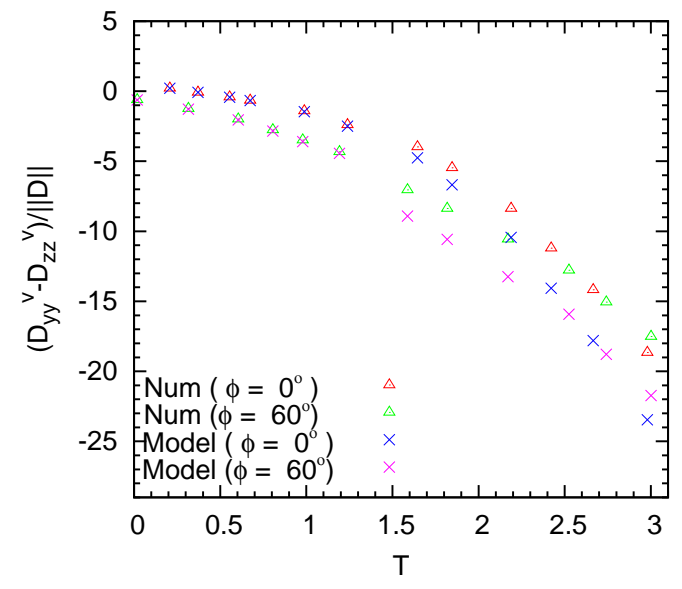

(b) $\left(D_{y y}^{v}-D_{z z}^{v}\right) /\|\mathbf{D}\|$

Fig. 15. Normalized void strain rates $\left(D_{x x}^{v}-D_{m}^{v}\right) /\|\mathbf{D}\|$ and $\left(D_{y y}^{v}-D_{z z}^{v}\right) /\|\mathbf{D}\|$ for an ellipsoidal void having $(a, b, c)=(10,2,1)$ and $f=0.01$, subjected to loadings having $\Sigma_{y y}=\Sigma_{z z} \neq \Sigma_{x x}$

two indices and symmetric in its last two ones. In fact this tensor possesses only three independent components (not counting those resulting from symmetries), $R_{x y x y}^{\mathrm{el}}, R_{x z x z}^{\mathrm{el}}$ and $R_{y z y z}^{\mathrm{el}}$. Indeed symmetry considerations imply that in elasticity, diagonal components of the overall strain rate tensor $\mathbf{D}$ in the principal basis of the void cannot generate any rotation of this void, while non-diagonal components representing shear can make the void rotate only within the shear plane.

In the plastic case, we propose, just like for the strain rate of the void, to heuristically replace the "elastic" equation (46) by

$$
\mathbf{\Omega}^{v}=\boldsymbol{\Omega}+\mathbf{R}: \mathbf{D} \quad \text { (plastic case) }
$$

where the new localization tensor $\mathbf{R}$ has the same symmetries and independent compo- 
nents as $\mathbf{R}^{\mathrm{el}}$, given by

$$
R_{x y x y} \equiv k_{x y} R_{x y x y}^{\mathrm{el}} \quad ; \quad R_{x z x z} \equiv k_{x z} R_{x z x z}^{\mathrm{el}} \quad ; \quad R_{y z y z} \equiv k_{y z} R_{y z y z}^{\mathrm{el}},
$$

$k_{x y}, k_{x z}$ and $k_{y z}$ being correction factors to be determined.

The expression of the factors $k_{i j}$ will again be looked for in the form of an interpolation between the extremal values $k_{i j}^{\mathrm{sph}}, k_{i j}^{\mathrm{cyl}}$ and $k_{i j}^{\text {sand }}$ corresponding to voids having the shape of a sphere, a circular cylinder and an infinite planar empty layer:

$$
\left\{\begin{aligned}
k_{x y} & \equiv \lambda k_{x y}^{\mathrm{sph}}+\mu k_{x y}^{\mathrm{cyl}}+\nu k_{x y}^{\mathrm{sand}} \\
k_{x z} & \equiv \lambda k_{x z}^{\mathrm{sph}}+\mu k_{x z}^{\mathrm{cyl}}+\nu k_{x z}^{\mathrm{sand}} \\
k_{y z} & \equiv \lambda k_{y z}^{\mathrm{sph}}+\mu k_{y z}^{\mathrm{cyl}}+\nu k_{y z}^{\mathrm{sand}}
\end{aligned}\right.
$$

with $\lambda \geq 0, \mu \geq 0, \nu \geq 0, \lambda+\mu+\nu=1$. Note that the interpolation coefficients used here are again the same for all coefficients $k_{i j}$.

The determination of correction coefficients will fortunately require much less work for the rotation rate of the void than for its strain rate. Indeed:

- For a spherical void, one may choose the directions $x, y, z$ parallel to the principal directions of $\mathbf{D}$. This tensor is then diagonal in the chosen principal basis of the void so that, by the remark made above, it cannot generate any rotation of this void. Thus the localization tensor $\mathbf{R}^{\mathrm{el}}$ is zero, which permits to take

$$
k_{x y}^{\mathrm{sph}}=k_{x z}^{\mathrm{sph}}=k_{y z}^{\mathrm{sph}}=1 .
$$

- For a circular cylindrical void, rotational symmetry implies equivalence of the necessary corrections on the expressions of $\Omega_{x y}^{v}$ and $\Omega_{x z}^{v}$. Also, in elasticity the projection of $\Omega^{v}$ onto the plane $y z$ is necessarily zero, as is easily seen by taking the directions $y$ and $z$ parallel to the principal directions of the projection of $\mathbf{D}$ onto this plane. This implies that the projection of the tensor $\mathbf{R}^{\mathrm{el}}$ onto the plane $y z$ is zero and insensitive to corrections. These elements suggest to take

$$
k_{x y}^{\mathrm{cyl}}=k_{x z}^{\mathrm{cyl}} \quad ; \quad k_{y z}^{\mathrm{cyl}}=1 .
$$

- Finally in the sandwich case, the same argument as in Subsection 2.3 shows that the elastic localization tensor $\mathbf{R}^{\mathrm{el}}$ applies irrespective of the constitutive law, so that again it requires no correction:

$$
k_{x y}^{\text {sand }}=k_{x z}^{\text {sand }}=k_{y z}^{\text {sand }}=1 .
$$

Thus a single coefficient need be determined, $k_{x y}^{\text {cyl }}{ }^{5}$

$\overline{5}$ This conclusion (like that concerning the determination of a single coefficient $h^{\text {sph }}$ for the strain rate of a spherical void) holds only within the framework of approximations made. For instance, in elasticity, a circular cylindrical void subjected to simultaneous shear strain rates $D_{x y}$ and $D_{y z}$ necessarily has $\Omega_{y z}^{v}=0$, but this does not remain true in plasticity, so that $R_{y z x y} \neq 0$ and/or $R_{y z y z} \neq 0$ in general; thus the complete determination of the rotation rate of such a void 


\subsection{Correction coefficient for the circular cylindrical case}

Using the expressions of the tensors $\boldsymbol{\Pi}$ and $\mathbf{L}^{\mathrm{el}}$ for the circular cylindrical geometry and equations $(46)_{2}$ and (48), one gets for the $x y$ component of the tensorial equation (47), in the absence of any overall rotation $\Omega$ :

$$
\Omega_{x y}^{v}=\frac{1-f}{1+f} k_{x y}^{\mathrm{cyl}} D_{x y} \quad \text { (plastic case). }
$$

The numerical simulations used to determine the coefficient $h_{44}^{\text {cyl }}$ can also serve to determine $k_{x y}^{\mathrm{cyl}}$, using equation (53) with the values of $\Omega_{x y}^{v}$ and $D_{x y}$ obtained numerically. Figure 16 , analogous to Figure 5 for $h_{44}^{\text {cyl }}$, shows the results obtained. Just like $h_{44}^{\text {cyl }}, k_{x y}^{\text {cyl depends }}$ on the deviatoric transverse invariant $X$ defined by equation (31) and also to some extent on the ratio $\Sigma_{y y} / \Sigma_{x x}$. A reasonable "average" representation of the numerical results disregarding this extra dependence is provided by the approximate formula

$$
k_{x y}^{\mathrm{cyl}}=1+11(1-\sqrt{f})^{11}-(1-\sqrt{f})^{11} \frac{170 X^{2}}{1+20 X^{2}} .
$$

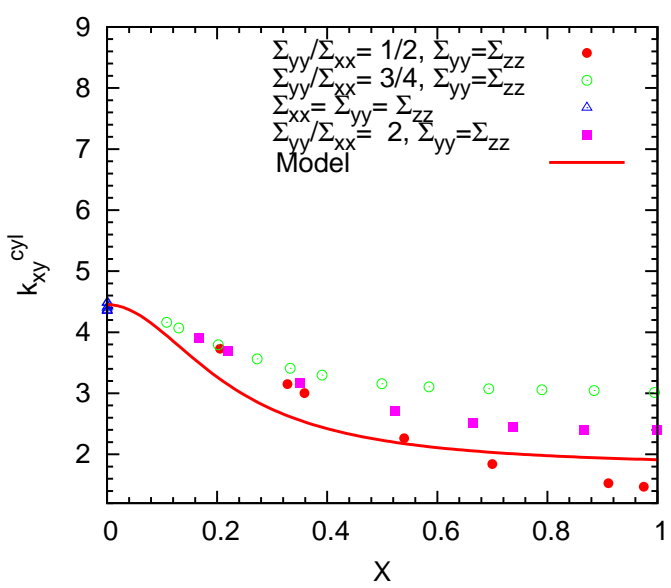

(a) $f=0.01$

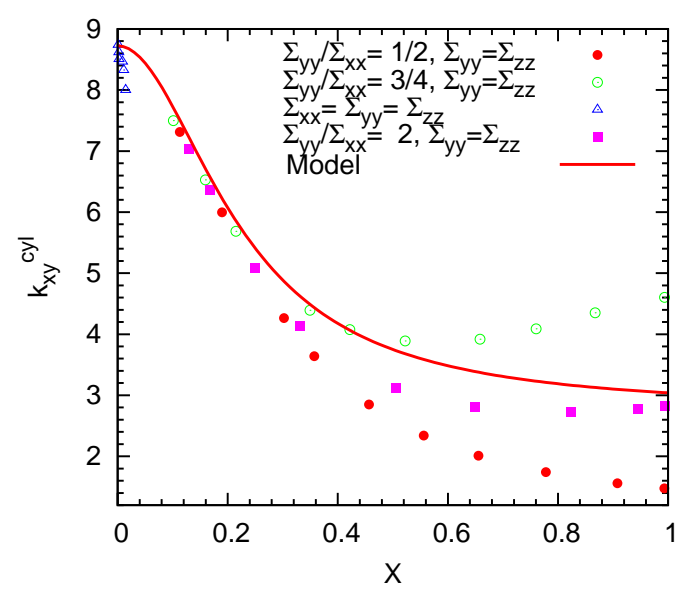

(b) $f=0.001$

Fig. 16. The coefficient $k_{x y}^{\text {cyl }}$ as a function of $X$, for $f=0.01$ and $f=0.001$

\subsection{Correction coefficients for the spheroidal and general ellipsoidal cases}

To get the coefficients $k_{i j}$ in other cases, we adopt exactly the same interpolation scheme as for the coefficients $h_{\alpha \beta}$. By equations (50), (51) and (52), this leads to the following expressions:

- for a prolate spheroidal void,

$$
k_{x y}^{\mathrm{prol}}\left(f, e_{x z} ; \boldsymbol{\Sigma}\right)=1-e_{x z}^{m}+e_{x z}^{m} k_{x y}^{\mathrm{cyl}}(f ; \boldsymbol{\Sigma}) \quad ; \quad k_{x z}^{\mathrm{prol}}=k_{x y}^{\mathrm{prol}} \quad ; \quad k_{y z}^{\mathrm{prol}}=1 ;
$$

would require that of these components of $\mathbf{R}$ and thus go beyond the determination of a single coefficient correcting the elastic law (46). 
- for an oblate spheroidal void,

$$
k_{x y}^{\mathrm{obl}}=k_{x z}^{\mathrm{obl}}=k_{y z}^{\mathrm{obl}}=1
$$

- for a general ellipsoidal void,

$$
k_{x y}\left(f, k, e_{x z} ; \boldsymbol{\Sigma}\right)=(1-k) k_{x y}^{\mathrm{prol}}\left(f, e_{x z} ; \boldsymbol{\Sigma}\right)+k \quad ; \quad k_{x z}=k_{x y} \quad ; \quad k_{y z}=1 .
$$

Testing these expressions numerically in general would require considering non-diagonal tensors $\boldsymbol{\Sigma}$ in the principal basis of the void, which would make it necessary to mesh more than $1 / 8$ of the ellipsoidal cell and result in extremely heavy simulations; therefore tests are limited to special cases requiring only modest meshes, see Subsection 4.4 below.

A final remark is that recent finite element micromechanical calculations performed by Scheyvaerts et al. (2011) led these authors to state that Kailasam and Ponte-Castaneda (1998)'s uncorrected elastic equation was sufficient to describe the rotation of the void in the plastic case. This statement seems to be in conflict with the conclusions of the present work. The explanation of Scheyvaerts et al. (2011)'s assertion is that they considered only the three cases of a spherical and two spheroidal, prolate and oblate voids with the same aspect ratio, $a / c=3$, in which the required correction is indeed minor according to our own findings; indeed equations (50), (55) and (56) predict no plastic correction for the spherical and oblate spheroidal voids, and a moderate one for the prolate spheroidal void which has $e_{x z} \simeq 0.943$ and $e_{x z}^{30} \simeq 0.171$, implying a value of $k_{x y}^{\text {prol }}$ rather close to $k_{x y}^{\text {sph }}=1$. But had Scheyvaerts et al. (2011) studied other cases, they would have concluded that the necessary correction may be large; see Figure 16 providing values of $k_{x y}$ for a circular cylindrical void.

\section{$4.4 \quad$ Numerical tests for elliptic cylindrical voids}

Equation $(57)_{3}$ shows that as a result of the interpolation procedure adopted, the correction coefficient $k_{y z}$ pertaining to the rotation of the void in the plane perpendicular to its major axis is postulated to be unity. This postulate may be put to test by numerically studying the rotation of elliptic cylindrical voids subjected to an in-plane shear strain rate $D_{y z}$; the choice of a cylindrical geometry reduces the necessary mesh to reasonable proportions, and that of a non-circular cross section warrants that the rotation rate $\Omega_{y z}^{v}$ is nonzero. The $y z$ component of the tensorial equation (47), combined with equation $(48)_{3}$, yields

$$
\Omega_{y z}^{v}=2 k_{y z} R_{y z y z}^{\mathrm{el}} D_{y z}
$$

where the component $R_{y z y z}^{\mathrm{el}}$ is to be deduced from equation $(46)_{2}$, using the values of $\boldsymbol{\Pi}$ and $\mathbf{L}^{\mathrm{el}}$ for the geometry considered. This equation permits to calculate the coefficient $k_{y z}$ using the numerical values of $\Omega_{y z}^{v}$ and $D_{y z}$.

In practice, we consider two elliptic cylindrical voids having $(a, b, c)=(+\infty, 5,1)$ and $(+\infty, 15,1)$ respectively, and $f=0.01$; calculations are performed in plane strain $\left(D_{x x}=\right.$ 0 ), imposing a shear strain rate $D_{y z}$, plus equal lateral strain rates $D_{y y}, D_{z z}$ in order to vary the lateral triaxiality $T_{h}$ defined by equation (28). Figure 17 shows the coefficient $k_{y z}$ 
obtained for the two voids, as a function of this triaxiality. For the void having $b / c=15$, the coefficient does not depart too much from unity, as expected for this geometry rather close to a sandwich for which no correction is required, see Subsection 4.1 above. However, for the void having $b / c=5, k_{y z}$ differs more from unity. It is interesting in particular that this coefficient changes sign when $T_{h}$ increases; this means that for a given overall strain rate $D_{y z}$, the void rotates in opposite directions for low and large lateral triaxialities. This counterintuitive phenomenon, which does not seem to have been noticed before, is a kind of equivalent of the BHS effect, but for the rotation rate of the void instead of its strain rate.

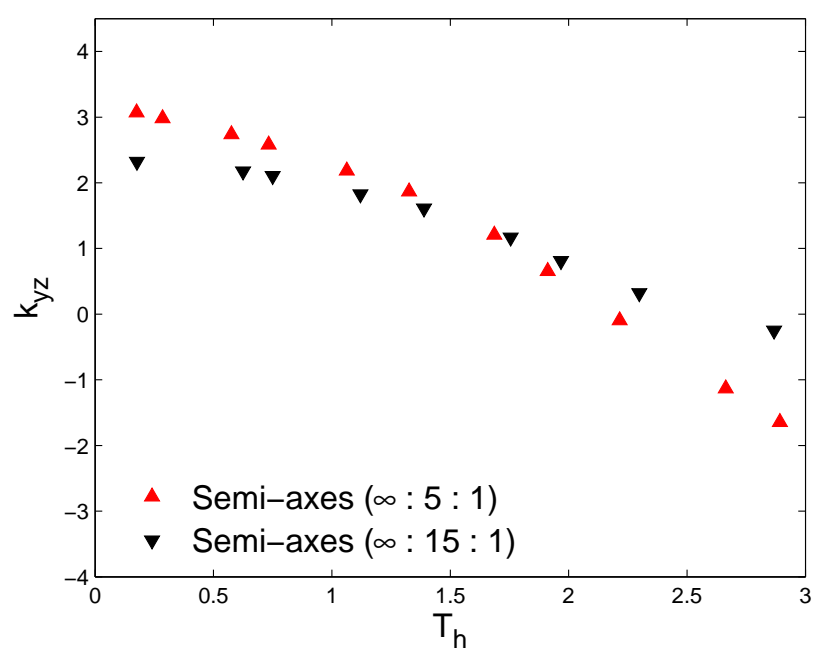

Fig. 17. The coefficient $k_{y z}$ as a function of $T_{h}$ for elliptic cylindrical voids, for $f=0.01$

Since a unity value for $k_{y z}$ is unavoidable within the framework of approximations adopted, we have no choice but to neglect this phenomenon. The resulting error is comparable to those made when neglecting the non-collinearity of the deviators of $\mathbf{D}^{v}$ and $\mathbf{D}$ for a spherical void, or when disregarding the dependence of the coefficient $k_{x y}^{\mathrm{cyl}}$ on quantities other than $X$, see Subsections 3.2 and 4.2.

\section{Evolution of the length and orientation of the axes of ellipsoidal voids}

The tensors $\mathbf{D}^{v}$ and $\Omega^{v}$ provide the strain and rotation rates of the void. However the true quantities of interest are the strain and rotation rates of its axes, which are different because these axes are not material directions. These rates may be related to $\mathbf{D}^{v}$ and $\boldsymbol{\Omega}^{v}$, see (Aravas and Ponte-Castaneda, 2004), whose results are recalled in Appendix B for completeness. But the expression of the rotation rate of the axes involves fractions with the denominators $a^{2}-b^{2}, b^{2}-c^{2}, c^{2}-a^{2}$, which diverge when two semi-axes of the void become equal. Although this feature does not raise any particular theoretical problem, it is bound to generate difficulties in the numerical implementation of the model. For this reason, we shall not use Aravas and Ponte-Castaneda (2004)'s expressions of the strain and rotation rates of the axes of the void, but look instead for the evolution equation of the quadratic form $\mathcal{P}$ characterizing its ellipsoidal shape. This evolution equation will be seen to be free of any such inconvenient divergence. 
Let $O$ denote the center of a given void, $M$ a current point on its surface and $\mathbf{u} \equiv \mathbf{O M}$ the separation vector between these points. The equation of the void surface reads

$$
\mathcal{P}(\mathbf{u}) \equiv \frac{\left(\mathbf{u}_{\mathbf{e}} \mathbf{e}_{x}\right)^{2}}{a^{2}}+\frac{\left(\mathbf{u} \mathbf{e}_{y}\right)^{2}}{b^{2}}+\frac{\left({\left.\mathbf{u} . \mathbf{e}_{z}\right)^{2}}_{c^{2}}\right.}{c^{2}}=1
$$

where $\mathbf{e}_{x}, \mathbf{e}_{y}, \mathbf{e}_{z}$ denote unit vectors parallel to the principal directions $x, y, z$ of the void. Clearly, the eigenvalues of the matrix $\mathbf{P} \equiv\left(P_{i j}\right)_{1 \leq i, j \leq 3}$ of the quadratic form $\mathcal{P}$ in the fixed frame of the observer are $a^{-2}, b^{-2}, c^{-2}$, and the associated eigenvectors are $\mathbf{e}_{x}, \mathbf{e}_{y}$, $\mathbf{e}_{z}$.

When the void moves, its strain rate is regarded as uniform, ${ }^{6}$ so that the material point $O$ remains at its center; and of course the material point $M$ remains on its surface. Therefore $\mathcal{P}(\mathbf{u})$ remains invariably equal to unity so that

$$
\frac{d}{d t}[\mathcal{P}(\mathbf{u})]=\frac{d}{d t}\left(P_{i j} u_{i} u_{j}\right)=\dot{P}_{i j} u_{i} u_{j}+P_{i j} \dot{u}_{i} u_{j}+P_{i j} u_{i} \dot{u}_{j}=0 .
$$

But since $\mathbf{u} \equiv \mathbf{O M}$ is a material vector, its derivative is given by the classical kinematic formula

$$
\dot{\mathbf{u}}=\left(\mathbf{D}^{v}+\Omega^{v}\right) \cdot \mathbf{u} \quad \Rightarrow \quad \dot{u}_{i}=\left(D_{i k}^{v}+\Omega_{i k}^{v}\right) u_{k} .
$$

Inserting this expression into the preceding equation, one gets

$$
\dot{P}_{i j} u_{i} u_{j}+P_{i j}\left(D_{i k}^{v}+\Omega_{i k}^{v}\right) u_{k} u_{j}+P_{i j} u_{i}\left(D_{j k}^{v}+\Omega_{j k}^{v}\right) u_{k}=0
$$

or equivalently, interchanging indices:

$$
\left[\dot{P}_{i j}+P_{k j}\left(D_{k i}^{v}+\Omega_{k i}^{v}\right)+P_{i k}\left(D_{k j}^{v}+\Omega_{k j}^{v}\right)\right] u_{i} u_{j}=0 .
$$

Since the vector $\mathbf{u}$ takes all directions of space when the point $M$ spans the surface of the void, this implies that

$$
\dot{P}_{i j}+P_{k j}\left(D_{k i}^{v}+\Omega_{k i}^{v}\right)+P_{i k}\left(D_{k j}^{v}+\Omega_{k j}^{v}\right)=0
$$

or equivalently

$$
\dot{\mathbf{P}}+\mathbf{P} \cdot\left(\mathbf{D}^{v}+\boldsymbol{\Omega}^{v}\right)+\left(\mathbf{D}^{v}+\boldsymbol{\Omega}^{v}\right)^{T} \cdot \mathbf{P}=\mathbf{0},
$$

which is the evolution equation of the matrix $\mathbf{P}$ looked for. This equation is clearly free of any divergence when two semi-axes of the void become equal.

Thus, in the numerical implementation of the model, to update the semi-axes and their orientations, one may avoid the use of the possibly divergent expressions of the strain and rotation rates of the void axes. One may use instead equation (60) to update the quadratic form characterizing the ellipsoidal geometry of the void. The semi-axes of the void and their orientations may then be obtained at each instant through diagonalization of the $3 \times 3$ symmetric matrix $\mathbf{P}$ of this quadratic form in the observer's fixed frame, using elementary computer routines. (In the case of equal eigenvalues, the issue of indetermination

$\overline{6}$ This property holds rigorously for an infinite and elastic medium (Eshelby, 1957), and approximately for a finite and plastic one. 
of principal directions of course persists, but the problem is solved in standard routines through some arbitrary choice of these directions).

\section{Summary of equations proposed}

- Definition of geometric quantities : the semi-axes of the void are denoted $a, b$ and $c$ with $a \geq b \geq c$, and the corresponding directions, $x, y$ and $z$. The eccentricity $e_{x z}$ of the void in the $x z$ plane and Madou and Leblond (2012a,b)'s parameter $k$ are defined by equations (33) and (42). The porosity is denoted $f$.

- Definition of mechanical quantities : the triaxiality $T$, Lode parameter $\omega$, lateral triaxiality $T_{h}$ and deviatoric transverse invariant $X$ are defined by equations (19), (28) and (31).

- Elastic localization tensors : the tensors $\mathbf{L}^{\mathrm{el}}$ and $\mathbf{R}^{\mathrm{el}}$ are given by equations $(1)_{2}$ and $(46)_{2}$. The components of the matrix $\overline{\mathbf{L}}^{\mathrm{el}}$ may be deduced from those of the tensor $\mathbf{L}^{\mathrm{el}}$ through equations (7).

- Correction coefficients for the matrix $\overline{\mathbf{L}}^{\mathrm{el}}$ : the matrix $\mathbf{h}$ of these coefficients is given by equations (44) and (45), $h_{22}^{\mathrm{prol}}, h_{33}^{\mathrm{prol}}, h_{44}^{\mathrm{prol}}$ and $h^{\mathrm{obl}}$ being themselves provided by equations (36) and (40) where $h^{\text {sph }}, h_{33}^{\text {cyl }}$ and $h_{44}^{\text {cyl }}$ are given by equations $(25),(29)$ and (32).

- Correction coefficients for the tensor $\mathbf{R}^{\mathrm{el}}$ : the coefficients $k_{x y}, k_{x z}$ and $k_{y z}$ are given by equation (57), $k_{x y}^{\text {prol }}$ being itself provided by equation (55) where $k_{x y}^{\text {cyl }}$ is given by equation (54).

- Plastic localization tensors : the matrix $\overline{\mathbf{L}}$ and the tensor $\mathbf{R}$ are related to their elastic counterparts and the correction factors through equations (9) and (48). The components of the tensor $\mathbf{L}$ may be deduced from those of the matrix $\overline{\mathbf{L}}$ through equations (8).

- Strain and rotation rates of the void: equations (2) and (47) relate the tensors $\mathbf{D}^{v}$ and $\boldsymbol{\Omega}^{v}$ to the overall strain and rotation rates $\mathbf{D}$ and $\boldsymbol{\Omega}$ through the localization tensors $\mathbf{L}$ and $\mathbf{R}$.

- Evolution of the semi-axes of the void and their orientations : the evolution of the matrix $\mathbf{P}$ of the quadratic form characterizing the ellipsoidal geometry of the void is governed by the values of $\mathbf{D}^{v}$ and $\boldsymbol{\Omega}^{v}$ through equation (60). The semi-axes $a, b, c$ of the void and the corresponding directions $x, y, z$ may be obtained at each instant through diagonalization of this matrix.

\section{Conclusion}

The aim of this paper was to propose good evolution equations for the length and orientation of the axes of a general ellipsoidal void embedded in a plastic matrix. These equations may be used to calculate the evolution of internal parameters of constitutive models for plastic porous materials accounting for void shape effects.

In the approach adopted, a central role was played by expressions of the strain and rotation rates of an ellipsoidal void embedded in an elastic matrix proposed by Ponte-Castaneda 
and Zaidman (1994) and Kailasam and Ponte-Castaneda (1998) from homogenization theory. It was however proposed to correct these expressions through introduction of heuristic coefficients determined numerically in a number of "reference cases" and suitably interpolated between these cases; the aim of these corrections being to approximately account for plastic effects, the importance of which has been known since the seminal work of Budiansky et al. (1982).

In the first reference case of a spherical void, a complete numerical study of the void strain rate as a function of the loading state was possible. The observation was made, apparently for the first time, that in plasticity the deviators of the void strain rate and the overall strain rate are not collinear in general, in contrast with Ponte-Castaneda and Zaidman (1994)'s expression of the void strain rate, even in its corrected form. Disregarding this non-collinearity, we determined the (unique) correction factor required in this case as a function of the porosity, the triaxiality and the Lode parameter, all of which were found to significantly influence it.

In the second reference case of a circular cylindrical void, a similar complete study of the void strain rate was impossible because of the excessive variety of load cases to be considered; the study was therefore limited to a number of hopefully significant cases. The two correction coefficients necessary in this case were determined as a function of the porosity, the lateral triaxiality and some transverse deviatoric invariant of the overall stress tensor.

The third reference case of a "sandwich" made of two planar sound layers surrounding an empty one did not necessitate any numerical study, since Ponte-Castaneda and Zaidman (1994)'s expression of the void strain rate was observed not to require any correction then.

Interpolation formulae were then proposed to calculate the correction coefficients for the void strain rate for arbitrary void geometries. This was done by considering (i) a prolate spheroidal void as intermediary between spherical and circular cylindrical ones; (ii) an oblate spheroidal void as intermediary between a spherical one and an infinite planar empty layer; and (iii) a general ellipsoidal void as intermediary between prolate and oblate spheroidal ones. The formulae proposed were validated numerically for two spheroidal, prolate and oblate voids, and two general ellipsoidal ones.

The definition of the correction coefficients for the void rotation rate was envisaged next. This required much less numerical work than for the void strain rate, because Kailasam and Ponte-Castaneda (1998)'s "elastic" expression was observed not to require any correction in the first and third reference cases, and introduction of a single correction coefficient in the second one.

It was then proposed, to calculate the evolution of the length and orientation of the axes of the void, to determine the evolution of the quadratic form characterizing its ellipsoidal shape, which was shown to be governed by a simple equation involving its strain and rotation rates. The semi-axes of the void and their orientations may then be calculated at each instant through diagonalization of this quadratic form. This procedure avoids direct use of the evolution equations of the length and orientation of the axes, which involve numerically cumbersome divergences when two semi-axes become equal. 
In the future, Madou and Leblond (2012a,b)'s criterion for plastic porous materials containing general ellipsoidal voids, completed by the evolution equations of its internal parameters defined here, will be implemented into some finite element code. As a first application, the behavior of an elementary porous cell, treated as a single finite element obeying the homogenized model, will be studied for various void shapes and loading conditions. Special attention will be devoted to the case of low triaxialities, where void shape effects are expected to be maximal. Reference will be made to a number of micromechanical finite element simulations performed by Tvergaard and coworkers under such loading conditions (see Dahl et al. (2012); Nielsen et al. (2012); Tvergaard (2012) for most recent accounts), which evidenced a mechanism of damage in shear arising from the sole change of the void shape, without any increase of the porosity. Our hope is to reproduce such a damage mechanism using Madou and Leblond (2012a,b)'s homogenized model. It is probable though that this will require completing it through incorporation of extra elements like possible contact between the crack faces and void coalescence, which played a decisive role in the micromechanical simulations of Tvergaard and coworkers.

Another future line of work will consist in extending Madou and Leblond (2012a,b)'s model for ellipsoidal voids embedded in an isotropic von Mises matrix, to similar voids embedded in an anisotropic Hill matrix. Reference will be made to the works of Keralavarma and Benzerga (2008) and Monchiet et al. (2008) who have achieved, in the case of spheroidal voids, an extension of this type of the GLD model. These authors showed that use, in a limit-analysis of a spheroidal porous cell made of some Hill material, of the same trial velocity fields as for a von Mises matrix, led to a reasonable estimate of the macroscopic yield criterion for moderate material anisotropies. It is hoped that use of the same trick will again yield acceptable results in the case of ellipsoidal voids.

\section{References}

Agoras M., Ponte Castaneda P. (2013). Iterated linear comparison bounds for viscoplastic porous materials with "ellipsoidal" microstructures. J. Mech. Phys. Solids, 61, 701-725.

Aravas N., Ponte Castaneda P. (2004). Numerical methods for porous metals with deformation-induced anisotropy. Comput. Methods Appl. Mech. Engrg., 193, 3767-3805.

Budiansky B., Hutchinson J.W., Slutsky S. (1982). Void growth and collapse in viscous solids. In: Mechanics of Solids, the Rodney Hill Anniversary Volume, H.G. Hopkins and M.J. Sewell, eds., Pergamon Press, Oxford, pp. 13-45.

Dahl J., Nielsen K.L., Tvergaard V. (2012). Effect of contact conditions on void coalescence at low stress triaxiality shearing. ASME J. Appl. Mech., 79, 021003-1 to 7.

Danas K., Ponte-Castaneda P. (2008a). A finite-strain model for anisotropic viscoplastic porous media: I - Theory. Eur. J. Mech. A/Solids, 28, 387-401.

Danas K., Ponte-Castaneda P. (2008b). A finite-strain model for anisotropic viscoplastic porous media: II - Applications. Eur. J. Mech. A/Solids, 28, 402-416.

Eshelby J.D. (1957). The determination of the elastic field of an ellipsoidal inclusion, and related problems. Proc. Roy. Soc. London A, 241, 376-396.

Fleck N., Hutchinson J. (1986). Void growth in shear. Proc. Roy. Soc. London A, 407, 435-458. 
Gologanu M. (1997). Etude de quelques problèmes de rupture ductile des métaux. Ph.D. Thesis, Université Pierre et Marie Curie (Paris VI) (in French).

Gologanu M., Leblond J.B., Devaux J. (1993). Approximate models for ductile metals containing non-spherical voids - Case of axisymmetric prolate ellipsoidal cavities. $J$. Mech. Phys. Solids, 41, 1723-1754.

Gologanu M., Leblond J.B., Devaux J. (1994). Approximate models for ductile metals containing non-spherical voids - Case of axisymmetric oblate ellipsoidal cavities. ASME J. Engng. Materials Technol., 116, 290-297.

Gologanu M., Leblond J.B., Perrin G., Devaux J. (1997). Recent extensions of Gurson's model for porous ductile metals. In: Continuum Micromechanics, P. Suquet, ed., Springer-Verlag, New-York, pp. 61-130.

Gurson A.L. (1977). Continuum theory of ductile rupture by void nucleation and growth: Part I - Yield criteria and flow rules for porous ductile media. ASME J. Engng. Materials Technol., 99, 2-15.

Huang Y. (1991). Accurate dilatation rates for spherical voids in triaxial stress fields. ASME J. Appl. Mech., 58, 1084-1086.

Kailasam M., Ponte-Castaneda P. (1998). A general constitutive theory for linear and nonlinear particulate media with microstructure evolution. J. Mech. Phys. Solids, 46, 427-465.

Keralavarma S.M., Benzerga A. (2008). An approximate yield criterion for anisotropic porous media. Comptes-Rendus Mécanique, 336, 685-749.

Koplik J., Needleman A. (1988). Void growth and coalescence in porous plastic solids. Int. J. Solids Structures, 24, 835-853.

Leblond J.B., Gologanu M. (2008). External estimate of the yield surface of an arbitrary ellipsoid containing a confocal void. Comptes-Rendus Mécanique, 336, 813-819.

Leblond J.B., Perrin G., Suquet P. (1994). Exact results and approximate models for porous viscoplastic solids. Int. J. Plasticity, 10, 213-235.

Lee B., Mear M. (1992). Axisymmetric deformation of power-law solids containing a dilute concentration of aligned spheroidal voids. J. Mech. Phys. Solids, 40, 1805-1836.

Madou K., Leblond J.B. (2012a). A Gurson-type criterion for porous ductile solids containing arbitrary ellipsoidal voids - I: Limit-analysis of some representative cell. Journal of the Mechanics and Physics of Solids, 60, 1020-1036.

Madou K., Leblond J.B. (2012b). A Gurson-type criterion for porous ductile solids containing arbitrary ellipsoidal voids - II: Determination of yield criterion parameters. Journal of the Mechanics and Physics of Solids, 60, 1037-1058.

Monchiet V., Cazacu O., Charkaluk E., Kondo D. (2008). Macroscopic yield criteria for plastic anisotropic materials containing spheroidal voids. Int. J. Plasticity, 24, 11581189 .

Nielsen K.L., Dahl J., Tvergaard V. (2012). Collapse and coalescence of spherical voids subject to intense shearing: studied in full 3D. Int. J. Fracture, 177, 97-108.

Ponte-Castaneda P., Zaidman M. (1994). Constitutive models for porous materials with evolving microstructure. J. Mech. Phys. Solids, 42, 1459-1492.

Press W.H., Teukolsky S., Vetterling W., Flannery B. (2007). Numerical Recipes, Third Edition, Cambridge University Press.

Rice J., Tracey D. (1969). On the ductile enlargement of voids in triaxial stress fields. J. Mech. Phys. Solids, 17, 201-217.

Scheyvaerts F., Onck P., Tekoglu C., Pardoen T. (2011). The growth and coalescence 
of ellipsoidal voids in plane strain under combined shear and tension. J. Mech. Phys. Solids, 59, 373-397.

Tvergaard V. (2012). Effect of stress state and spacing on voids in a shear field. Int. J. Solids Structures, 49, 3047-3054.

Yee K., Mear M. (1996). Effect of void shape on the macroscopic response of non-linear solids. Int. J. Plast., 12, 45-68. 


\section{A Appendix: components of Eshelby's tensors $\mathrm{S}$ and $\Pi$}

The nonzero components of Eshelby (1957)'s first and second tensors $\mathbf{S}$ and $\boldsymbol{\Pi}$ are given, for an incompressible material, by

$$
\left\{\begin{array}{rl}
S_{x x x x} & \equiv \frac{3}{4 \pi} a^{2} I_{a a} \\
S_{x x y y} & \equiv \frac{3}{4 \pi} b^{2} I_{a b} \\
S_{x y x y} & \equiv \frac{3}{8 \pi}\left(a^{2}+b^{2}\right) I_{a b}
\end{array} \quad ; \quad \Pi_{x y x y} \equiv \Pi_{x y y x} \equiv-\Pi_{y x x y} \equiv-\Pi_{y x y x} \equiv \frac{1}{8 \pi}\left(I_{b}-I_{a}\right)\right.
$$

plus similar formulae resulting from simultaneous cyclic interchange of $x, y, z$ and $a, b, c$. In these expressions $I_{a}, I_{a a}, I_{a b}$, etc. are Eshelby (1957)'s integrals defined by

$$
\left\{\begin{aligned}
I_{a} & \equiv 2 \pi a b c \int_{0}^{+\infty} \frac{d \rho}{\left(a^{2}+\rho\right) v(\rho)} \\
I_{a a} & \equiv 2 \pi a b c \int_{0}^{+\infty} \frac{d \rho}{\left(a^{2}+\rho\right)^{2} v(\rho)} \quad, \quad v(\rho) \equiv \sqrt{\left(a^{2}+\rho\right)\left(b^{2}+\rho\right)\left(c^{2}+\rho\right)} \\
I_{a b} & \equiv \frac{2 \pi}{3} a b c \int_{0}^{+\infty} \frac{d \rho}{\left(a^{2}+\rho\right)\left(b^{2}+\rho\right) v(\rho)}
\end{aligned}\right.
$$

and similar formulae. The practical (numerical) calculation of these integrals, based on formulae of Eshelby (1957) and computer routines provided in Press et al. (2007)'s book, is straightforward and explained in detail in Appendix C of (Madou and Leblond, 2012b).

\section{B Appendix: rates of the principal axes and directions of the void}

The evolution equations of the semi-axes $a, b, c$ of the ellipsoidal void and the corresponding unit vectors $\mathbf{e}_{x}, \mathbf{e}_{y}, \mathbf{e}_{z}$ read, in terms of the components of the strain and rotation rates $\mathbf{D}^{v}, \Omega^{v}$ in the void's principal basis (Aravas and Ponte-Castaneda, 2004):

$$
\frac{\dot{a}}{a}=D_{x x}^{v} \quad ; \quad \frac{\dot{b}}{b}=D_{y y}^{v} \quad ; \quad \frac{\dot{c}}{c}=D_{z z}^{v}
$$

and

$$
\left\{\begin{array}{l}
\dot{\mathbf{e}}_{x}=\left(\frac{a^{2}+b^{2}}{a^{2}-b^{2}} D_{y x}^{v}+\Omega_{y x}^{v}\right) \mathbf{e}_{y}+\left(\frac{a^{2}+c^{2}}{a^{2}-c^{2}} D_{z x}^{v}+\Omega_{z x}^{v}\right) \mathbf{e}_{z} \\
\dot{\mathbf{e}}_{y}=\left(\frac{b^{2}+c^{2}}{b^{2}-c^{2}} D_{z y}^{v}+\Omega_{z y}^{v}\right) \mathbf{e}_{z}+\left(\frac{b^{2}+a^{2}}{b^{2}-a^{2}} D_{x y}^{v}+\Omega_{x y}^{v}\right) \mathbf{e}_{x} \\
\dot{\mathbf{e}}_{z}=\left(\frac{c^{2}+a^{2}}{c^{2}-a^{2}} D_{x z}^{v}+\Omega_{x z}^{v}\right) \mathbf{e}_{x}+\left(\frac{c^{2}+b^{2}}{c^{2}-b^{2}} D_{y z}^{v}+\Omega_{y z}^{v}\right) \mathbf{e}_{y} .
\end{array}\right.
$$

